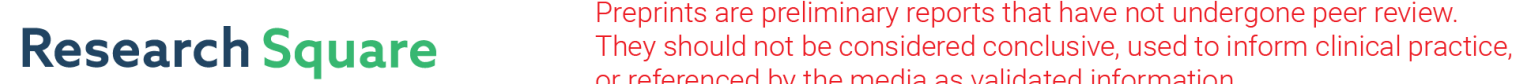 or referenced by the media as validated information. \\ CLAMPs allow single cell tracking of KRASG12C inhibition and endow druggability to KRAS mutants
}

Marie Evangelista ( $\square$ evangem2@gene.com )

Genentech

Chris Davies

Genentech

Angela Oh

Genentech

Rana Mroue

Genentech

Melinda Mulvihil

Genentech

Yang Xiao

Genentech

Siyu Feng

Genentech

Sangeeta Jayakar

Genentech

Emily Chan

Genentech

Vidhyalakshmi Arumugam

Genentech

Cheng Lu

Genentech

Yvonne Franke

Genentech

Mark Merchant

Genentech

Hartmut Koeppen

Genentech Inc.

John Quinn

Genentech Inc.

Sushant Malhotra

Genentech Inc.

Hans Purkey 
Genentech Inc.

Joachim Rudolph

Genentech Inc.

James Koerber

Genentech Inc. https://orcid.org/0000-0001-7756-972X

\section{Weiru Wang}

Genentech Inc.

\section{Article}

Keywords: KRASG12C, SWII pocket, CLAMPs

Posted Date: May 13th, 2021

DOI: https://doi.org/10.21203/rs.3.rs-133893/v1

License: (c) (i) This work is licensed under a Creative Commons Attribution 4.0 International License. Read Full License

Version of Record: A version of this preprint was published at Nature Biotechnology on January 6th, 2022. See the published version at https://doi.org/10.1038/s41587-021-01126-9. 


\section{CLAMPs allow single cell tracking of KRAS ${ }^{\mathrm{G} 12 \mathrm{C}}$ inhibition and endow druggability to KRAS mutants}

Authors: Christopher W. Davies ${ }^{1,+}$, Angela $\mathrm{Oh}^{2,+}$, Rana Mroue ${ }^{3,+}$, Melinda M. Mulvihill', Yang Xiao ${ }^{3}$, Siyu Feng ${ }^{3}$, Sangeeta Jayakar ${ }^{5}$, Emily Chan ${ }^{6}$, Vidhyalakshmi Arumugam ${ }^{6}$, Cheng Lư ${ }^{7}$, Yvonne Franke ${ }^{8}$, Mark Merchant ${ }^{6}$, Harmut Koeppen ${ }^{5}$, John G. Quinn ${ }^{4}$, Sushant Malhotra ${ }^{9}$, Hans E. Purkey ${ }^{9}$, Joachim Rudolph ${ }^{9}$, James T. Koerber ${ }^{1, *}$, Weiru Wang ${ }^{2, *}$, Marie Evangelista ${ }^{3, *}$

\section{Affiliations:}

${ }^{1}$ Department of Antibody Engineering, Genentech, Inc., South San Francisco, CA 94080

$10 \quad{ }^{2}$ Department of Structural Biology, Genentech, Inc., South San Francisco, CA 94080

${ }^{3}$ Department of Discovery Oncology, Genentech, Inc., South San Francisco, CA 94080

${ }^{4}$ Department of Biochemical and Cellular Pharmacology, Genentech, Inc., South San Francisco, CA 94080

${ }^{5}$ Department of Pathology, Genentech, Inc., South San Francisco, CA 94080

${ }^{6}$ Department of Translational Oncology, Genentech, Inc., South San Francisco, CA 94080

$15 \quad{ }^{7}$ Department of Protein Chemistry, Genentech, Inc., South San Francisco, CA 94080

${ }^{8}$ Department of BioMolecular Resources, Genentech, Inc., South San Francisco, CA 94080

${ }^{9}$ Department of Discovery Chemistry, Genentech, Inc., South San Francisco, CA 94080

${ }^{+}$Equal contributions

*Correspondence to: evangelista.marie@gene.com, wang.weiru@gene.com, koerber.james@gene.com 


\begin{abstract}
The discovery of covalent inhibitors binding the switch II (SWII) pocket has enabled therapeutic intervention in $K R A S^{G I 2 C}$ driven tumors and represents a milestone in targeting KRAS-driven cancers. However, the transient nature and high energetic barrier required for binding this pocket has been an obstacle in successfully targeting other KRAS mutant oncoproteins. We report the discovery of KRAS Conformation Locking Antibodies for Molecular Probe discovery (CLAMP)s that specifically recognize the unique conformation of KRAS ${ }^{\mathrm{G} 12 \mathrm{C}}$ induced by covalent inhibitors. KRAS CLAMPs enable single cell resolution of covalent inhibitor-bound KRAS ${ }^{\mathrm{G} 12 \mathrm{C}}$ in cells and in vivo tumor models, providing a biomarker for direct target engagement of KRAS ${ }^{\mathrm{G} 12 \mathrm{C}}$ inhibition. KRAS
\end{abstract} CLAMPs bind multiple KRAS mutants and stabilize an open conformation of the SWII pocket increasing the affinity of weak non-covalent SWII pocket ligands. This work provides new insights into KRAS ${ }^{\mathrm{G} 12 \mathrm{C}}$ upon treatment with covalent inhibitors and offers a path towards targeting the SWII pocket in other RAS mutants. 
Main: RAS proteins are small, membrane-bound guanine nucleotide-binding proteins encoded by three genes (HRAS, NRAS and KRAS). RAS proteins act as molecular switches by cycling between active GTP-bound and inactive GDP-bound conformations ${ }^{1}$. The active GTP-bound conformation allows RAS to signal to a diverse set of downstream effectors including RAF, PI3K, and RAL GDS ${ }^{2-11}$ and oncogenic mutations in RAS, frequently at position 12 , reduce GTP hydrolysis resulting in constitutively active RAS signaling ${ }^{12-15}$. The picomolar affinity for GTP or GDP, in addition to the lack of obvious pockets for small molecule binding in RAS have hampered drug discovery efforts against oncogenic mutant RAS for several decades.

The landmark discovery of $\mathrm{KRAS}^{\mathrm{G} 12 \mathrm{C}}$ inhibitors that covalently modify the mutant Cys12 residue has provided a novel and promising opportunity for drugging $\mathrm{KRAS}^{\mathrm{G} 12 \mathrm{C}}$ mutant tumors ${ }^{16}$. Compound 12, ARS-853, ARS-1620, AIM-4, and clinical molecules AMG 510 and MRTX849 bind and stabilize an "open" conformation in the switch II (SWII) region not previously observed in KRAS-GDP or KRAS-GTP ${ }^{16-23}$. The mechanism of action of such SWII pocket covalent binders is through stabilization of this transient pocket via initial binding to the pocket followed by chemical reaction with Cys $12{ }^{17}$. This modification irreversibly locks KRAS ${ }^{\mathrm{G} 12 \mathrm{C}}$ in a GDP-bound inactive state by preventing intrinsic or SOS-mediated exchange, causes tumor growth inhibition in pre-clinical models, and is showing promising clinical activity ${ }^{17,18}$. Discovery of these KRAS ${ }^{\mathrm{G} 12 \mathrm{C}}$ inhibitors relied heavily on the covalent reactivity with Cys 12 to inhibit $\mathrm{KRAS}^{\mathrm{G} 12 \mathrm{C}}$ protein ${ }^{24}$. Thus, the viability of strategies targeting this pocket in other KRAS mutants lacking this critical mutant cysteine residue remains to be determined.

Despite these successes, the consequence of covalent inactivation of $\mathrm{KRAS}^{\mathrm{G} 12 \mathrm{C}}$ has not been investigated due to the lack of reagents capable of visualizing endogenous KRAS ${ }^{\mathrm{G} 12 \mathrm{C}}$ covalent modification in tumor cells. Such 20 reagents could be powerful tools to investigate the dynamics and homogeneity of $\mathrm{KRAS}^{\mathrm{G} 12 \mathrm{C}}$ covalent modification upon inhibitor treatment in individual cell populations, which may shed light on how tumors cells acquire resistance to $\mathrm{KRAS}^{\mathrm{G} 12 \mathrm{C}}$ inhibitors.

Synthetic monoclonal antibodies (mAbs) represent an emerging tool to stabilize unique protein conformations and the ability to combine in vitro selections with conformationally-locked targets has enabled the 25 discovery of novel conformational sensors against RAS, caspases, and GPCRs ${ }^{25-28}$. These antibodies have tremendous potential to elucidate the biological role of protein conformations in cells. They may also have utility in drug discovery efforts by serving as structural chaperones to improve structure-based drug design and/or by stabilizing rare protein conformations to increase the success of lead finding efforts.

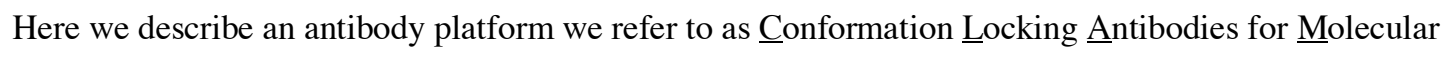
Probe discovery (CLAMP)s. We applied this platform using covalently modified KRAS ${ }^{\mathrm{G} 12 \mathrm{C}}$ and discovered two classes of CLAMPs. Class I CLAMPs specifically recognize a conformational state in KRAS ${ }^{\mathrm{G} 12 \mathrm{C}}$ associated with covalent ligand bound to the SWII pocket. One such CLAMP (1A5) enables detection of covalently modified KRAS $^{\mathrm{G} 12 \mathrm{C}}$ within individual cancer cells and tumors, providing a direct biomarker for KRAS ${ }^{\mathrm{G} 12 \mathrm{C}}$-inhibitor mediated target engagement, and can be coupled with RAS pathway markers to assess pathway inhibition and subsequent rebound at the cellular level. Class II CLAMPs also exhibit affinity for KRAS ${ }^{\mathrm{G}_{12}}$-GDP alone and stabilize an open conformation of the SWII pocket in the absence of ligand. This feature significantly improves the affinity of multiple non-covalent inhibitors for KRAS ${ }^{\mathrm{G} 12 \mathrm{C}}$ and $\mathrm{KRAS}^{\mathrm{WT}}$. Intriguingly, one class II CLAMP (2H11), bound 
multiple KRAS mutants including $\mathrm{KRAS}^{\mathrm{G} 12 \mathrm{~V}}{ }_{-} \mathrm{GDP}, \mathrm{KRAS}^{\mathrm{G} 12 \mathrm{R}}$-GDP, and KRAS ${ }^{\mathrm{Q} 6 \mathrm{H}_{-}}$GDP. We propose that KRAS CLAMPs are an important biology and drug discovery tool for investigating KRAS ${ }^{\mathrm{G} 12 \mathrm{C}}$ covalent modification in vivo and will provide a unique platform for identifying novel chemical matter targeting the KRAS SWII pocket. We envisage that general application of CLAMPs may enable identification of ligands for otherwise intractable drug targets.

\section{Discovery and characterization of KRAS SWII pocket CLAMPs}

We developed a method to identify KRAS CLAMPs using an in vitro selection strategy with synthetic antibody libraries. We applied this method to KRAS, which exhibits conformational heterogeneity in its switch I (SWI) and SWII regions, to identify antibodies that would stabilize an "open" SWII pocket conformation compatible with that induced by KRAS ${ }^{\mathrm{G} 12 \mathrm{C}}$ covalent inhibitors (Fig. 1A). To identify KRAS CLAMPs, we leveraged distinct conformations of KRAS ${ }^{\mathrm{G} 12 \mathrm{C}}$ : unmodified $\mathrm{KRAS}^{\mathrm{G} 12 \mathrm{C}}$-GDP, KRAS ${ }^{\mathrm{G} 12 \mathrm{C}}$-GDP with a covalent inhibitor, and KRAS ${ }^{\mathrm{G} 12 \mathrm{C}}$-GMPPCP (non-hydrolysable GTP mimetic) (Fig. 1B). Four rounds of biopanning were performed in which the synthetic phage libraries were incubated in solution with biotinylated KRAS ${ }^{\mathrm{G} 12 \mathrm{C}}$-GDP covalently modified with GNE-1952, a tool G12C inhibitor ${ }^{29}$ (Fig. S1A). In order to drive selections towards the unique conformation of covalently inhibited $\mathrm{KRAS}^{\mathrm{G} 12 \mathrm{C}}$-GDP, selections were done in the presence of excess of $\mathrm{KRAS}^{\mathrm{G} 12 \mathrm{C}}$-GDP and $\mathrm{KRAS}^{\mathrm{G} 12 \mathrm{C}}$-GMPPCP in solution. To enable the discovery of antibodies capable of stabilizing the open SWII pocket conformation in the absence of inhibitor, we also performed three rounds of selection with

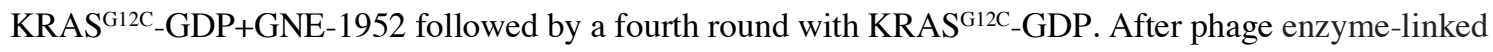
immunosorbent assay (ELISA) screens to confirm specificity, we generated IgGs for eleven unique clones and characterized their binding specificity by ELISA (Fig. 1C) and surface plasmon resonance (SPR) (Fig. 1D, Fig. S1B, and Table S1).

The eleven antibodies were assayed for their ability to recognize covalently modified KRAS ${ }^{\mathrm{G} 12 \mathrm{C}}$ with additional KRAS ${ }^{\mathrm{G} 12 \mathrm{C}}$ covalent molecules ARS-853 and ARS-1620 ${ }^{17,19}$ (Fig. S1A) to filter out CLAMPs that only 25 recognize GNE-1952 bound KRAS ${ }^{\mathrm{G} 12 \mathrm{C}}$-GDP. All antibodies showed affinity to KRAS ${ }^{\mathrm{G} 12 \mathrm{C}}$-GDP covalently modified by GNE-1952, ranging from 1-139 nM (Table S1). However, one group of antibodies (five clones) was specific for the GNE-1952-bound KRAS ${ }^{\mathrm{G} 12 \mathrm{C}_{-} \text {GDP }}$ conformation while a second group of antibodies (six clones)

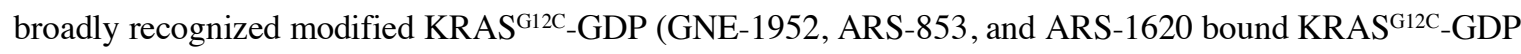
conformations) and were referred to as KRAS CLAMPs since they appeared to be conformation rather than chemotype specific. Epitope mapping analysis revealed that these two groups bound distinct but partially

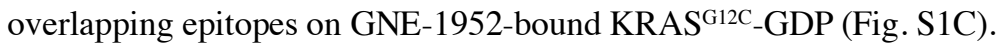

The six KRAS CLAMPs could be further divided into two classes: the Class I CLAMP, 1A5, had high specificity for covalently bound KRAS ${ }^{\mathrm{G} 12 \mathrm{C}}$-GDP with $>100$-fold improved affinity for this target compared to KRAS $^{\mathrm{G} 12 \mathrm{C}}$-GDP, whereas the Class II CLAMPs, (1E5, 2H11, 2A3, 3A12, and 4G12) recognized KRAS ${ }^{\mathrm{G} 12 \mathrm{C}}$-GDP independent of inhibitor presence, as measured by ELISA and SPR (Fig. 1C, Fig. 1D and Table S1). To further establish the antibody specificity in cells, we performed an immunoprecipitation experiment with 1A5 and 2H11 on lysates from HCC1171 KRAS ${ }^{G 12 C}$ mutant cells treated with ARS-1620 or DMSO control. We found that both Class I 
and II CLAMPs specifically immunoprecipitated covalently bound KRAS ${ }^{\mathrm{G} 12 \mathrm{C}_{-} \mathrm{GDP}}$ but not KRAS ${ }^{\mathrm{G} 12 \mathrm{C}_{-}}$GDP alone (Fig. 1E) suggesting that the affinity of the CLAMPs was insufficient to bind to KRAS ${ }^{\mathrm{G} 12 C_{-}}$GDP or KRAS ${ }^{\mathrm{G} 12 \mathrm{C}_{-}}$GTP in cells. To further explore the potential of these antibodies, we focused on representative class I (1A5) and class II (2H11) antibodies.

5

\section{Tracking $\operatorname{KRAS}^{\mathrm{G} 12 \mathrm{C}}$ covalent modification in cells}

Since the 1A5 CLAMP showed the highest specificity for covalently modified KRAS ${ }^{\text {G12C }}$-GDP compared to $\mathrm{KRAS}^{\mathrm{G} 12 \mathrm{C}}$-GDP, we tested whether the 1A5 CLAMP could specifically detect inhibitor-bound KRAS ${ }^{\mathrm{G} 12 \mathrm{C}}$ in cells. Immunofluorescence (IF) staining with the 1A5 CLAMP resulted in detection of covalently modified KRAS ${ }^{\mathrm{G} 12 \mathrm{C}}$ in HCC1171 KRAS ${ }^{G 12 C}$-mutant cells treated with a variety of KRAS ${ }^{\mathrm{G} 12 \mathrm{C}}$ covalent inhibitors, including GNE-1952, ARS-853, ARS-1620, and AMG 510 (Fig. 2A, 2B), thus confirming the ability of 1A5 to recognize a common conformation induced by various KRAS ${ }^{\mathrm{G} 12 \mathrm{C}}$ covalent inhibitors. In contrast, there was no detectable 1A5 CLAMP staining in HCC1171 KRAS ${ }^{G I 2 C}$-mutant cells treated with DMSO or in HCT116 KRAS ${ }^{G l 3 D}$-mutant cells treated with GNE-1952 (Fig. S2A). The intensity of 1A5 CLAMP staining in ARS-1620-treated HCC1171 cells appeared to be dose- and time-dependent (Fig. 2B, 2C) and was consistent with results from immunoblotting for covalently inhibited KRAS ${ }^{\mathrm{G} 12 \mathrm{C}}$ (as measured both by an upward electrophoretic mobility shift of the KRAS ${ }^{\mathrm{G} 12 \mathrm{C}}$ protein band migration) and by inhibition of RAS pathway markers pERK and pMEK in a bulk population of cells (Fig. S2B, lane 1,2). Studies by IF revealed that ARS-1620-induced covalent modification of KRAS ${ }^{\mathrm{G} 12 \mathrm{C}}$ in cells occurred in a fairly synchronous fashion occurring at both the plasma membrane as well as in punctate compartments of cells (Fig. 2A-C). Since antibodies specific for RAS-GDP do not exist, staining with the 1A5 CLAMP likely provides information on the localization of KRAS ${ }^{\mathrm{G} 12 \mathrm{C}}$-GDP in cells upon or shortly after covalent modification. Moreover, the uniformity and kinetics of 1A5 staining indicates that covalent modification of KRAS ${ }^{\mathrm{G} 12 \mathrm{C}}$ may occur independent of cell cycle stage. Since covalent modification of KRAS ${ }^{\mathrm{G} 12 \mathrm{C}}$-GDP is dependent on intrinsic GTP hydrolysis (16-17), these findings additionally suggest that intrinsic KRAS ${ }^{G 12 C}$-GTP hydrolysis is also not cell cycle dependent (Fig. 2B, 2C). The 1A5 CLAMP was also used to visualize covalent modification of KRAS ${ }^{\mathrm{G} 12 \mathrm{C}}$-GDP by ARS-1620 in a number of $K R A S^{G I 2 C}$ lines expressing very low levels of KRAS ${ }^{\mathrm{G} 12 \mathrm{C}}$ protein (Fig. 2D) highlighting the sensitivity of $1 \mathrm{~A} 5$ in detecting covalently modified KRAS ${ }^{\mathrm{G} 12 \mathrm{C}}-\mathrm{GDP}$.

We next evaluated the utility of combining the 1A5 CLAMP and pS6 antibody to simultaneously measure KRAS $^{\mathrm{G} 12 \mathrm{C}}$ covalent modification and RAS signaling in individual cells using flow cytometry. Similar to the IF

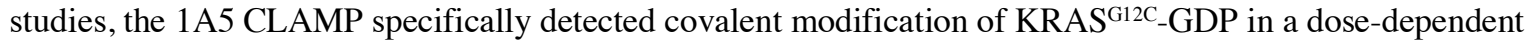
manner (Fig. 2E). This staining correlated with an observed dose-dependent decrease in pS6 levels in most of the population (Fig. 2E). Interestingly, a small population ( 1\%) of $1 \mathrm{~A}^{+}$cells remained high for pS6 suggesting this population of cells maintain RAS signaling despite covalent modification of KRAS ${ }^{\mathrm{G} 12 \mathrm{C}}$.

We also surveyed a set of commercially available antibodies to KRAS to determine their conformational specificity. We identified two antibodies (Abcam and Ras10) that had comparable affinity to KRAS ${ }^{\mathrm{G} 12 \mathrm{C}}$, with or without covalent modification, by immunoprecipitation and ELISA (Fig. 3A and 3B), indicating that these antibodies are not specific to the conformation induced by covalent modification. In contrast, the iDab antibody, 
which was reported to be highly specific for HRAS-GTP, showed little to no binding to covalently modified

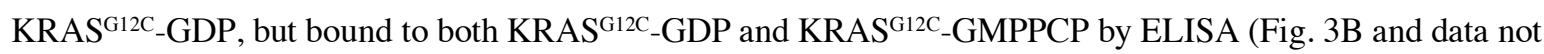
shown), and in immunoprecipitation studies (Fig. 3A) ${ }^{27}$. Since the iDab antibody binds an epitope that spans both the SWI and SWII regions, the SWII conformation induced by covalent modification of KRAS ${ }^{\mathrm{G} 12 \mathrm{C}}$-GDP likely prevents iDab binding.

Given the complementary specificities of the 1A5 CLAMP and iDab, we wondered if the 1A5 CLAMP and iDab antibodies could be used in combination to co-stain and visualize both unmodified and covalently modified KRAS $^{\mathrm{G} 12 \mathrm{C}}$ within the same cell. We conducted IF experiments with both 1A5 CLAMP and iDab antibodies on HCC1171 KRAS ${ }^{G 12 C}$ cells treated with a dose titration of ARS-1620. Similar to previous IF experiments with 1A5, we detected an increase in 1A5 staining that correlated with high concentrations of ARS-1620 treatment. Furthermore, the increased 1A5 staining coincided with decreased staining with the iDab antibody, confirming that these two antibodies stain different KRAS ${ }^{\mathrm{G} 12 \mathrm{C}}$ conformations (Fig. 3C). Co-staining with both antibodies allowed us to also monitor the re-synthesis of KRAS ${ }^{\mathrm{G} 12 \mathrm{C}}$. Treatment of KRAS ${ }^{\mathrm{G} 12 \mathrm{C}}$ cells with ARS-1620 or ARS-853 for 16 hours resulted in almost complete covalent modification of KRAS ${ }^{\mathrm{G} 12 \mathrm{C}}$ based on the appearance of $1 \mathrm{~A} 5$ and disappearance of iDab staining respectively by IF and was consistent with an electromobility shift of KRAS based on immunoblot analysis (Fig. 3D and Fig. S2B). Upon drug washout, the appearance of unmodified KRAS ${ }^{\mathrm{G} 12 \mathrm{C}}$ was apparent as early as 6 hours and was increased at 24 hours as evident by immunoblot analysis (Fig. S2B). Similar to immunoblot analysis, 24 hour drug washout studies also showed a significant decrease in 1A5 staining and an increase in iDab antibody staining by IF (Fig. 3D).

Tumor cell adaptation to ARS-1620 has been attributed to new synthesis of KRAS ${ }^{\mathrm{G} 12 \mathrm{C}}$ protein not bound to drug and subsequent reactivation of pERK, a marker of RAS pathway activity ${ }^{30}$. As such we investigated whether the 1A5 staining signal decreased over time due to new synthesis of KRASG12C that remains unmodified, and whether that decrease correlated with the appearance of a pERK signal. We first treated NCI-H358 KRAS ${ }^{\mathrm{G} 12 \mathrm{C}}$ mutant cells with $1 \mu \mathrm{M}$ ARS-1620 for various time points $(4,24,48,72$ and 96 hours) to assess both target occupancy, and pERK inhibition and potential rebound. In cells treated with $100 \mathrm{nM}$ and $1 \mu \mathrm{M}$ ARS-1620, we observed an increase of alkylated KRAS ${ }^{\mathrm{G} 12 \mathrm{C}}$ evident by the enhanced intensity of $1 \mathrm{~A} 5$ signal within the first 48 hours, followed by a drop between 72 to 96 hours, suggesting accumulation of unstained unmodified KRASG12C. Correspondingly, pERK intensity demonstrated a quick inhibition as early as 4 hours followed by a sharp rebound after $72 \mathrm{hrs} \mathrm{suggesting} \mathrm{that} \mathrm{the} \mathrm{rebound} \mathrm{of} \mathrm{pERK} \mathrm{is} \mathrm{likely} \mathrm{due} \mathrm{to} \mathrm{insufficient} \mathrm{target} \mathrm{occupancy} \mathrm{by} \mathrm{ARS-1620.} \mathrm{We}$ then treated cells with $300 \mathrm{nM}$ MRTX849 (a sufficiently more potent KRAS ${ }^{\mathrm{G} 12 \mathrm{C}}$ covalent inhibitors) at the clinically relevant dose. Unlike ARS-1620, treatment with MRTX849 resulted in a long-lasting increase in 1A5 signal intensity, which correlated with sustained suppression of pERK signaling 96 hours post-treatment. This study not only demonstrates that potent KRAS ${ }^{\mathrm{G} 12 \mathrm{C}}$ covalent inhibitors overcome pERK rebound in $\mathrm{KRAS}^{\mathrm{G} 12 \mathrm{C}}$ mutant tumors but also serves as proof-of-concept for using CLAMPS to study long-term drug dynamics and acquired resistance to therapy. 
We next sought to determine if the 1A5 CLAMP could detect covalently modified KRAS ${ }^{\text {G12C }}$ in human tumor xenograft experiments. Detection of covalently inhibited KRAS ${ }^{\mathrm{G} 12 \mathrm{C}}$ with the 1A5 CLAMP by immunohistochemistry (IHC) using formalin-fixed, paraffin-embedded (FFPE) tissue was not possible, likely due to the harsh formalin treatment that destroys the conformational epitope recognized by 1 A5. However, ARS-1620 covalently modified KRAS ${ }^{\mathrm{G} 12 \mathrm{C}}$ was readily detectable by IHC in tumor samples prepared as unfixed fresh frozen (FP) tissues (Fig. 4A). In addition, there appeared to be a subtle trend towards stronger staining in samples treated with $200 \mathrm{mg} / \mathrm{kg}$ ARS-1620 compared to samples treated with $50 \mathrm{mg} / \mathrm{kg}$ ARS-1620 (Fig. 4A). Tumors expressing lower amounts of KRAS ${ }^{G 12 C}$ covalently modified by ARS-1620 were also detected by the 1A5 CLAMP (Fig. 4B). Similar to in vitro cell experiments, the 1A5 CLAMP detected KRAS ${ }^{\mathrm{G} 12 \mathrm{C}}$ that was covalently modified by ARS1620 in FACS experiments with ex-vivo tumor samples and could be combined with the pS6 (Fig. 4C). Thus, these results show that the 1A5 CLAMP enables measurement of direct target engagement of KRAS ${ }^{\mathrm{G} 12 \mathrm{C}}$ inhibitors in KRAS $^{\mathrm{G} 12 \mathrm{C}}$ tumor samples and enables single cell analysis with markers of RAS pathway activation.

\section{KRAS CLAMPs improve the non-covalent affinity of SWII pocket ligands to KRAS ${ }^{\text {G12C }}$ and KRAS ${ }^{\text {WT }}$}

We hypothesized that the 2H11 class II CLAMP might stabilize the open conformation of the SWII pocket in KRAS ${ }^{\mathrm{G} 12 \mathrm{C}}$-GDP based on its ability to recognize both the unbound and covalently modified conformation of KRAS $^{\mathrm{G} 12 \mathrm{C}}$. Such CLAMPs would then be predicted to improve the weak non-covalent affinity for ligands that bind in the SWII pocket. To test this possibility, we developed an SPR assay to specifically detect binding to the SWII pocket using a SWII-blocked reference (see Materials and Methods). We measured the affinity of various SWII pocket covalent ligands (GNE-1952, ARS-853, ARS-1620, and a non-covalent version of GNE-1952 that lacked the reactive acrylamide moiety) (Fig. S1A) to KRAS ${ }^{\mathrm{G} 12 \mathrm{C}}$-GDP in the presence and absence of 2H11 CLAMP. Additionally, we also included KRAS ${ }^{\text {WT }}$-GDP to determine if $2 \mathrm{H} 11$ could stabilize the SWII pocket in other KRAS variants. Impressively, we found that inhibitor affinity was greatly enhanced in the presence of the 2H11 CLAMP (Fig. 5A and Fig. S3A). The 2H11 CLAMP increased the affinity of chemically diverse KRAS ${ }^{\mathrm{G} 12 \mathrm{C}}$ SWII ligands further confirming that it is not biased towards one particular chemotype suggesting that it stabilizes an open conformation of the SWII pocket in the absence of ligand.

\section{Structural analysis of CLAMP:KRAS ${ }^{\mathrm{G} 12 \mathrm{C}}$ complex}

We determined the crystal structure of KRAS ${ }^{\mathrm{G} 12 \mathrm{C}}$-GDP in complex with $2 \mathrm{H} 11$ fragment antigen-binding (Fab) at 2.2 ̊ resolution (Fig. 5B, upper panel) to gain further insights into the mode of action of the 2H11 CLAMP. Rather than binding to the KRAS ${ }^{\mathrm{G} 12 \mathrm{C}}$ SWII pocket, the $2 \mathrm{H} 11$ Fab contacts the outer surface of the SWII region to stabilize an open conformation of the pocket in an allosteric manner. The $2 \mathrm{H} 11$ Fab binding buries $\sim 745 \AA^{2}$ of total surface area and contacts residues from SWI, SWII and the center core $\beta$-sheet (Fig. 5B, lower panel). The 2H11 Fab complementarity-determining regions (CDRs) $\mathrm{H} 1$ and $\mathrm{H} 3$ contribute to the majority of the direct contacts with KRAS $^{\mathrm{G} 12 \mathrm{C}}$ (Fig. 5C). The long 13-residue CDR H3 loop directly engages the SWII region by inserting Trp99 into a small hydrophobic pocket, known as the DCAI pocket ${ }^{31,32}$ and is surrounded by $\mathrm{KRAS}^{\mathrm{G} 12 \mathrm{C}}$ residues Lys5, Leu6, Val7, Ser39, Asp54, Leu56, Tyr71, Thr74, Gly75 (Fig. 5B). Interestingly, 2H11 exploits this site with a chemically 
similar tryptophan side chain (Fig. S3B). CDR H1 contacts KRAS near the C-terminal end of SWI region and packs against a portion of the RAS-binding domain (RBD) binding site. Unlike iDab (26), 2H11 makes minimal contact with the residues in the N-terminal half of SWI (Fig. S3D), consistent with its observed ability to bind to both KRAS-GDP and GTP states. CDR L2 and H2 also contribute several van der Waals contacts with KRAS. CDR L2 contacts the N-terminal tip of the SWII helix, providing additional stabilization to the SWII loop but without overly restricting the SWII conformation. As shown in Figure 5D, the most flexible part of SWII, Gln60-Ala66, is completely free from direct contact with $2 \mathrm{H} 11$, maintaining flexibility in the pocket. Importantly, the SWII pocket lies in a stable open conformation that resembles the shape of previously published ligand bound KRAS structures suggesting easy accessibility for ligand binding ${ }^{23}$.

We then determined the crystal structure of $2 \mathrm{H} 11$ in complex with KRAS ${ }^{\mathrm{G} 12 \mathrm{C}}$ covalently modified by GNE1952 and compared it to $2 \mathrm{H} 11$ in complex with unbound $\mathrm{KRAS}^{\mathrm{G} 12 \mathrm{C}}$ (Fig. 5D) to test the above hypothesis. While, GNE-1952 caused a shift in the mainchain and sidechain conformation of the SWII residues (SWII C $\alpha$ atoms RMSD of 1.4Å) the remainder of KRAS and the $2 \mathrm{H} 11$ Fab CDRs structures remained largely identical. The sidechain of His95, which forms a hydrogen bond to the quinazoline nitrogen of the inhibitor, is the only residue outside SWII that changed conformation upon GNE-1952 binding. This interaction appears to be common for quinazoline scaffold compounds and is not affected by the presence of $2 \mathrm{H} 11 \mathrm{Fab}{ }^{17}$. To confirm $2 \mathrm{H} 11$ did not significantly alter the ligand binding mode, we determined the crystal structure of KRAS ${ }^{\mathrm{G} 12 \mathrm{C}}$-GDP covalently inhibited by GNE-1952 in the absence of the 2H11 Fab. Comparison of both inhibitor complex structures, with and without 2H11 Fab (Fig. S3C), revealed a highly similar ligand binding mode (Fig S3B) and atomic interactions,

20 although, in the absence of Fab, the SWII helix titled further inward to the pocket and the ligand displayed a concerted shift (SWII C $\alpha$ atoms RMSD of $0.87 \AA ̊$ ). This shift is likely influenced by crystal packing interactions and indicates that SWII maintains some level of flexibility upon ligand binding.

\section{Application of CLAMPs to other KRAS mutants}

Due to the dynamic nature of the SWII pocket, all previous efforts to discover novel compounds that bind this region have required the use of electrophilic functional groups that can form covalent bonds with Cys12. However, most compounds in screening libraries typically do not carry chemically reactive groups and thus, such libraries would be of limited use for screening against KRAS G12C. Based on our combined biochemical and structural results, we hypothesized that the 2H11 CLAMP could provide a novel tool for the discovery of new

30 ligands by enabling screens against the KRAS ${ }^{\mathrm{G} 12 \mathrm{C}}-2 \mathrm{H} 11$ complex in which the SWII pocket lies in an open conformation. Furthermore, the lack of convenient reactive residues in other oncogenic KRAS mutants has prevented the targeting of this region more generally. To explore whether $2 \mathrm{H} 11$ could also recognize the GDP bound states and potentially stabilize the open conformation of the SWII region in these mutants, we evaluated binding of 2H11 to a panel of KRAS mutants by ELISA (Fig. 5E). Quite strikingly, 2H11 exhibited strong binding

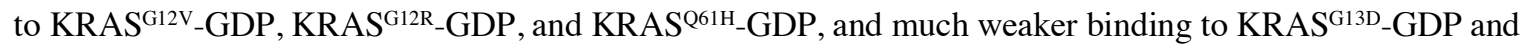
KRAS ${ }^{\text {WT }}$-GDP (Fig. 5E). The similar binding to G12V, G12R, and Q61H suggests that these mutants may adopt a shared SWI/SWII conformation that is distinct from G13D, G12C, and WT. Given that the 2H11 CLAMP binds 
multiple KRAS mutants and can increase the affinity of SWII pocket ligands, presumably by stabilizing the open conformation as we observe in our crystal structure, it may enable the identification of novel ligands against other RAS mutants.

\section{Discussion}

While RAS proteins are major oncogenic drivers of many cancers, the lack of stable pockets has hindered drug development. The recent discovery of KRAS ${ }^{\mathrm{G} 12 \mathrm{C}}$ covalent inhibitors revealed a cryptic pocket within the SWII region and sets a precedent for clinical activity for future ligands of mutant KRAS. Despite this success, whether other KRAS mutants can be targeted via the SWII pocket remains to be determined.

The discovery of KRAS CLAMPs have revealed new insights into KRAS covalent modification and can be used to visualize and track inhibitor-bound KRAS ${ }^{\mathrm{G} 12 \mathrm{C}}$ in cells and in vivo tumor models. Although the covalent modification of KRAS ${ }^{\mathrm{G} 12 \mathrm{C}}$ appeared to be homogenous in pre-clinical studies, modification of $\mathrm{KRAS}^{\mathrm{G} 12 \mathrm{C}}$ and subsequent tumor response in patient tumors is likely more heterogeneous. Implementation of a 1A5-based flow cytometry or IHC assay along with markers of KRAS activity pathway activity such as pERK in the clinical trials should enable rapid detection of covalent modification, confirming target engagement, and could also inform potential mechanisms of acquired resistance.

Biophysical and structural studies showed that the 2H11 CLAMP can induce a conformation with an open SWII pocket in KRAS ${ }^{\mathrm{G} 12 \mathrm{C}}$-GDP, can improve the affinity of SWII ligands, and may enable future efforts to target this pocket more broadly across KRAS mutants. While this strategy may be successful for other mutant proteins

20 with sufficient intrinsic GTP-hydrolysis; other KRAS mutants (e.g., KRAS $\left.{ }^{\mathrm{Q} 61 \mathrm{H}}\right)$ appear to be constitutively bound to GTP making it unclear whether targeting of these mutants is possible ${ }^{15}$. Recent work using a non-natural cysteine suggests that it is possible to identify compounds that engage the SWII pocket in both GDP and GTP bound states ${ }^{33}$. Future efforts to identify KRAS CLAMPs that also bind and stabilize the GTP state to promote a stable open SWII pocket irrespective of the nucleotide bound state may be possible and provide a foundation for small molecule screens using a CLAMP:KRAS mutant complex to expand the targeting of the SWII pocket to all RAS mutants.

Our CLAMP platform has led to the discovery of antibodies that recognize conformations that exist within the dynamic KRAS switch regions. The success of this strategy relied on the presence of a covalent ligand, highlighting the importance of locking conformationally dynamic proteins in different states to enable the discovery of unique conformation-specific antibodies. Additionally, we provide the first experimental evidence that CLAMPs can also induce and lock a conformation in the absence of ligand and thus, enable small molecule discovery efforts against transient pockets within conformationally dynamic proteins. More generally, our work has broad implications for antibody-assisted small molecule drug discovery against highly dynamic biological targets. 


\section{References}

1 Ostrem, J. M. \& Shokat, K. M. Direct small-molecule inhibitors of KRAS: from structural insights to mechanism-based design. Nat Rev Drug Discov 15, 771-785, doi:10.1038/nrd.2016.139 (2016).

2 Wood, K. W., Sarnecki, C., Roberts, T. M. \& Blenis, J. ras mediates nerve growth factor receptor modulation of three signal-transducing protein kinases: MAP kinase, Raf-1, and RSK. Cell 68, 1041-1050, doi:10.1016/0092-8674(92)90076-o (1992).

3 Howe, L. R. et al. Activation of the MAP kinase pathway by the protein kinase raf. Cell 71, 335-342, doi:10.1016/0092-8674(92)90361-f (1992).

$4 \quad$ Vojtek, A. B., Hollenberg, S. M. \& Cooper, J. A. Mammalian Ras interacts directly with the serine/threonine kinase Raf. Cell 74, 205-214, doi:10.1016/0092-8674(93)90307-c (1993).

5 Moodie, S. A., Willumsen, B. M., Weber, M. J. \& Wolfman, A. Complexes of Ras.GTP with Raf-1 and mitogen-activated protein kinase kinase. Science 260, 1658-1661, doi:10.1126/science.8503013 (1993).

6 Zhang, X. F. et al. Normal and oncogenic p21ras proteins bind to the amino-terminal regulatory domain of c-Raf-1. Nature 364, 308-313, doi:10.1038/364308a0 (1993).

7 Sjolander, A., Yamamoto, K., Huber, B.E. \& Lapetina, E. G. Association of p21 ras with phosphatidylinositol 3-kinase. Proc Natl Acad Sci U S A 88, 7908-7912, doi:10.1073/pnas.88.18.7908 (1991).

8 Rodriguez-Viciana, P. et al. Phosphatidylinositol-3-OH kinase as a direct target of Ras. Nature 370, 527532, doi:10.1038/370527a0 (1994).

9 Hofer, F., Fields, S., Schneider, C. \& Martin, G. S. Activated Ras interacts with the Ral guanine nucleotide dissociation stimulator. Proc Natl Acad Sci U S A 91, 11089-11093, doi:10.1073/pnas.91.23.11089 (1994).

10 Spaargaren, M. \& Bischoff, J. R. Identification of the guanine nucleotide dissociation stimulator for Ral as a putative effector molecule of R-ras, H-ras, K-ras, and Rap. Proc Natl Acad Sci U S A 91, 12609-12613, doi:10.1073/pnas.91.26.12609 (1994).

11 White, M. A., Vale, T., Camonis, J. H., Schaefer, E. \& Wigler, M. H. A role for the Ral guanine nucleotide dissociation stimulator in mediating Ras-induced transformation. J Biol Chem 271, 16439-16442, doi:10.1074/jbc.271.28.16439 (1996).

12 Fasano, O. et al. Analysis of the transforming potential of the human H-ras gene by random mutagenesis. Proc Natl Acad Sci U S A 81, 4008-4012, doi:10.1073/pnas.81.13.4008 (1984).

13 Gideon, P. et al. Mutational and kinetic analyses of the GTPase-activating protein (GAP)-p21 interaction: the C-terminal domain of GAP is not sufficient for full activity. Mol Cell Biol 12, 2050-2056, doi:10.1128/mcb.12.5.2050 (1992).

Scheffzek, K. et al. The Ras-RasGAP complex: structural basis for GTPase activation and its loss in oncogenic Ras mutants. Science 277, 333-338, doi:10.1126/science.277.5324.333 (1997).

Hunter, J. C. et al. Biochemical and Structural Analysis of Common Cancer-Associated KRAS Mutations. Mol Cancer Res 13, 1325-1335, doi:10.1158/1541-7786.MCR-15-0203 (2015).

16 Ostrem, J. M., Peters, U., Sos, M. L., Wells, J. A. \& Shokat, K. M. K-Ras(G12C) inhibitors allosterically control GTP affinity and effector interactions. Nature 503, 548-551, doi:10.1038/nature12796 (2013). 
Patricelli, M. P. et al. Selective Inhibition of Oncogenic KRAS Output with Small Molecules Targeting the Inactive State. Cancer Discov 6, 316-329, doi:10.1158/2159-8290.CD-15-1105 (2016).

Fakih, M. et al. Phase 1 study evaluating the safety, tolerability, pharmacokinetics (PK), and efficacy of AMG 510, a novel small molecule KRASG12C inhibitor, in advanced solid tumors. J Clin Oncol 37, 30033003, doi:10.1200/JCO.2019.37.15_suppl.3003 (2019).

Lito, P., Solomon, M., Li, L. S., Hansen, R. \& Rosen, N. Allele-specific inhibitors inactivate mutant KRAS G12C by a trapping mechanism. Science 351, 604-608, doi:10.1126/science.aad6204 (2016).

Zeng, M. et al. Potent and Selective Covalent Quinazoline Inhibitors of KRAS G12C. Cell Chem Biol 24, 1005-1016 e1003, doi:10.1016/j.chembiol.2017.06.017 (2017).

Canon, J. et al. The clinical KRAS(G12C) inhibitor AMG 510 drives anti-tumour immunity. Nature 575, 217-223, doi:10.1038/s41586-019-1694-1 (2019).

Hallin, J. et al. The KRAS(G12C) Inhibitor MRTX849 Provides Insight toward Therapeutic Susceptibility of KRAS-Mutant Cancers in Mouse Models and Patients. Cancer Discov 10, 54-71, doi:10.1158/21598290.CD-19-1167 (2020).

Janes, M. R. et al. Targeting KRAS Mutant Cancers with a Covalent G12C-Specific Inhibitor. Cell 172, 578589 e517, doi:10.1016/j.cell.2018.01.006 (2018).

Hansen, R. et al. The reactivity-driven biochemical mechanism of covalent KRAS(G12C) inhibitors. Nat Struct Mol Biol 25, 454-462, doi:10.1038/s41594-018-0061-5 (2018).

Nizak, C. et al. Recombinant antibodies to the small GTPase Rab6 as conformation sensors. Science 300, 984-987, doi:10.1126/science.1083911 (2003).

Gao, J., Sidhu, S. S. \& Wells, J. A. Two-state selection of conformation-specific antibodies. Proc Natl Acad Sci U S A 106, 3071-3076, doi:10.1073/pnas.0812952106 (2009).

Tanaka, T., Williams, R. L. \& Rabbitts, T. H. Tumour prevention by a single antibody domain targeting the interaction of signal transduction proteins with RAS. EMBO J 26, 3250-3259, doi:10.1038/sj.emboj.7601744 (2007).

28 McMahon, C. et al. Yeast surface display platform for rapid discovery of conformationally selective nanobodies. Nat Struct Mol Biol 25, 289-296, doi:10.1038/s41594-018-0028-6 (2018).

Li Liansheng, U. S. et al. INHIBITORS OF KRAS G12C I INHIBITEURS DE K-RAS G12C. WO patent WO2015054572A1 (2015).

Xue, J. Y. et al. Rapid non-uniform adaptation to conformation-specific KRAS(G12C) inhibition. Nature 577, 421-425, doi:10.1038/s41586-019-1884-x (2020).

Maurer, T. et al. Small-molecule ligands bind to a distinct pocket in Ras and inhibit SOS-mediated nucleotide exchange activity. Proc Natl Acad Sci U S A 109, 5299-5304, doi:10.1073/pnas.1116510109 (2012).

Sun, Q. et al. Discovery of small molecules that bind to K-Ras and inhibit Sos-mediated activation. Angew Chem Int Ed Engl 51, 6140-6143, doi:10.1002/anie.201201358 (2012).

Gentile, D. R. et al. Ras Binder Induces a Modified Switch-II Pocket in GTP and GDP States. Cell Chem Biol 24, 1455-1466 e1414, doi:10.1016/j.chembiol.2017.08.025 (2017). 
Lee, C. V. et al. High-affinity human antibodies from phage-displayed synthetic Fab libraries with a single framework scaffold. J Mol Biol 340, 1073-1093, doi:10.1016/j.jmb.2004.05.051 (2004).

35 Liang, W. C. et al. Function blocking antibodies to neuropilin-1 generated from a designed human synthetic antibody phage library. J Mol Biol 366, 815-829, doi:10.1016/j.jmb.2006.11.021 (2007).

Lombana, T. N., Dillon, M., Bevers, J., 3rd \& Spiess, C. Optimizing antibody expression by using the naturally occurring framework diversity in a live bacterial antibody display system. Sci Rep 5, 17488, doi:10.1038/srep17488 (2015).

37 Abdiche, Y. N. et al. High-throughput epitope binning assays on label-free array-based biosensors can yield exquisite epitope discrimination that facilitates the selection of monoclonal antibodies with functional activity. PLoS One 9, e92451, doi:10.1371/journal.pone.0092451 (2014).

38 Kabsch, W. Xds. Acta crystallographica. Section D, Biological crystallography 66, 125-132, doi:10.1107/S0907444909047337 (2010).

39 McCoy, A. J. et al. Phaser crystallographic software. Journal of applied crystallography 40, 658-674, doi:10.1107/S0021889807021206 (2007).

Emsley, P. \& Cowtan, K. Coot: model-building tools for molecular graphics. Acta crystallographica. Section D, Biological crystallography 60, 2126-2132, doi:10.1107/S0907444904019158 (2004).

41 Murshudov, G. N., Vagin, A. A. \& Dodson, E. J. Refinement of macromolecular structures by the maximumlikelihood method. Acta crystallographica. Section D, Biological crystallography 53, 240-255, doi:10.1107/S0907444996012255 (1997).

Adams, P. D. et al. PHENIX: a comprehensive Python-based system for macromolecular structure solution. Acta crystallographica. Section D, Biological crystallography $\mathbf{6 6}, \quad 213-221$, doi:10.1107/S0907444909052925 (2010).

Acknowledgments: We are thankful to Shiva Malek, Jim Kiefer, and Andrey Shaw for helpful discussions. We thank staff at Advanced Light Source and Stanford Synchrotron Radiation Light-source for their assistance on data collection.

Author contributions: Project conceptualization: J.T.K, W.W., M.E.; Protein production and labeling: A.O., C.L.; Antibody selections, SPR, and production: C.W.D., J.T.K.; Cell-based experiments: R.M., Y.X., S.F.; Advice and generation of small molecule inhibitors: J.R., S.M., H.P.; Construct generation: Y.F.; In vivo tumor studies: E.C., V.A., M.M.; Immunohistochemical analysis: S.J., H.K.; SPR with small molecules: M.M.M., J.Q.; Crystallography: A.O., W.W.; Structural analysis: W.W.; Manuscript writing: C.W.D., J.T.K., W.W., M.E.; J.T.K., W.W., and M.E. are co-senior authors. 
Figure Legends:

Figure 1: Discovery of KRAS CLAMPs.

(A) (Left) Overlay of RAS crystal structures reflect the conformational heterogeneity seen in the SWI (teal) and SWII regions (blue). (Right) Model of KRAS CLAMP stabilizing the "open" SWII pocket conformation that is also induced by $\mathrm{KRAS}^{\mathrm{G} 12 \mathrm{C}}$ covalent inhibitors.

(B) In vitro selection strategy to identify KRAS CLAMPs.

(C) ELISA characterization demonstrates that all eleven KRAS CLAMPs bind specifically to KRAS ${ }^{\mathrm{G} 12 \mathrm{C}}$-GDP + GNE-1952 with variable binding to KRAS ${ }^{\mathrm{G} 12 \mathrm{C}}$-GDP ( $\left.\mathrm{n}=3\right)$.

10 (D) SPR analysis confirms that both $1 \mathrm{~A} 5$ and $2 \mathrm{H} 11$ CLAMPs bind strongly to KRAS ${ }^{\mathrm{G} 12 \mathrm{C}}$-GDP when covalently modified with various covalent $\mathrm{KRAS}^{\mathrm{G} 12 \mathrm{C}}$ inhibitors.

(E) Both 1A5 and 2H11 CLAMPs selectively immunoprecipitate covalently modified KRAS ${ }^{\mathrm{G} 12 \mathrm{C}}$-GDP from cells treated with ARS-1620 but not KRAS ${ }^{\mathrm{G} 12 \mathrm{C}}$-GDP.

Figure 2: 1A5 CLAMP enables monitoring of $\mathrm{KRAS}^{\mathrm{G} 12 \mathrm{C}}$ covalent modification in multiple cellular assays. (A) 1A5 CLAMP stains HCC1171 KRAS ${ }^{G I 2 C}$ mutant cancer cells treated with various KRAS ${ }^{\mathrm{G} 12 \mathrm{C}}$ inhibitors $(5 \mu \mathrm{M}$ GNE-1952, $10 \mu$ M ARS-853, 37 nM AMG 510) and NCI-358 cells treated with 300 nM MRTX849 in immunofluorescence (IF) assays.

(B) IF staining with 1A5 CLAMP enables visualization of $\mathrm{KRAS}^{\mathrm{G} 12 \mathrm{C}}$ covalent modification kinetics over a drug dose range (0.001 to $10 \mu \mathrm{M})$, and reveals a time- and dose- dependent increase in covalent modification upon ARS1620 treatment in HCC1171 KRAS GI2C mutant cancer cells.

(C) Quantification of 1A5 CLAMP IF staining upon time-dependent covalent modification of HCC1171 KRAS cells treated with $1 \mu \mathrm{M}$ ARS-1620.

(D) $1 \mathrm{~A} 5$ detects covalently modified $\mathrm{KRAS}^{\mathrm{G} 12 \mathrm{C}}$ across three $K R A S^{G I 2 C}$ mutant cell lines with different expression 25 levels of KRAS ${ }^{\mathrm{GI} 2 \mathrm{C}}$.

(E) Multiplexed flow cytometry with $1 \mathrm{~A} 5$ and anti-pS6, a marker of KRAS pathway activation, reveals increased KRAS $^{\mathrm{G} 12 \mathrm{C}}$ covalent modification coincident with decreased pS6 in response to increasing concentrations of ARS853 in $\mathrm{HCC} 1171$ KRAS $^{G 12 C}$ cells.

Figure 3: A dual antibody assay using $1 \mathrm{A5}$ and a commercial antibody simultaneously tracks both unbound and covalently modified KRAS ${ }^{\mathrm{G} 12 \mathrm{C}}$.

(A) Differential immunoprecipitation of covalently modified and unbound $\mathrm{KRAS}^{\mathrm{G} 12 \mathrm{C}}$ with a panel of commercial antibodies identifies that iDab detects only unbound KRAS ${ }^{\mathrm{G} 12 C}$. Lysates were prepared from HCC1171 KRASGI2C cells treated with DMSO or ARS-1620.

35 (B) ELISA characterization of commercially available antibodies confirms that iDab selectively binds KRAS ${ }^{\mathrm{G}_{12 \mathrm{C}}}$ $\operatorname{GDP}(n=3)$. 
(C) 1A5 CLAMP and iDab enable the assessment of covalently modified and unbound KRAS ${ }^{\mathrm{G} 12 \mathrm{C}}$, respectively, by IF over a dose titration of ARS-1620. We observe a decrease in 1A5 staining as the concentration of ARS-1620 decreases and a parallel increase in iDab staining.

(D) Overlay of $1 \mathrm{~A} 5$ and iDab IF staining enables tracking of newly synthesized and unbound KRAS ${ }^{\mathrm{G} 12 \mathrm{C}}$ after ARS1620 washout, and confirms western blot results that covalently modified KRAS ${ }^{\mathrm{G} 12 \mathrm{C}}$ persists for at least $24 \mathrm{hr}$ postwashout.

(E) Overlay of 1A5 and pERK (Thr202/Tyr204) IF staining enables tracking of KRAS ${ }^{\mathrm{G} 12 \mathrm{C}}$ alkylation, inhibition, and rebound of MAPK signaling over time in NCI-H358 KRAS ${ }^{G I 2 C}$ mutant cells treated with covalent inhibitors (ARS1620 and MRTX849). Scale bar: $200 \mu \mathrm{m}$.

Figure 4: A KRAS CLAMP enables detection of covalently modified KRAS ${ }^{\text {G12C }}$ from high and low KRAS $^{\mathrm{G} 12 \mathrm{C}}$-expressing mouse xenografts treated with ARS-1620.

(A) Representative IHC images with KRAS 1A5 CLAMP on frozen sections of NCI-H358 (high KRAS ${ }^{G 12 C_{-}}$

15 expressing) xenografts in female C/B17 SCID mice following $8 \mathrm{hr}$ and 24hr treatment with the indicated concentrations of ARS-1620.

(B) Representative IHC images of NCI-H2122 (low KRAS $S^{G 12 C}$-expressing) xenografts in female CRL nude mice following $8 \mathrm{hr}$ treatment with the indicated concentrations of ARS-1620.

(C) Flow cytometry with 1A5 and anti-pS6 from dissociated NCI-H358 xenograft samples treated with ARS-1620

20 demonstrates a dose-dependent increase in $\mathrm{KRAS}^{\mathrm{G} 12 \mathrm{C}}$ covalent modification (bar graphs) that coincides with a decrease in pS6 (black circles) $(n=5)$.

\section{Figure 5 : $2 \mathrm{H11}$ CLAMP stabilizes the SWII pocket and binds multiple KRAS mutant proteins.}

25 (A) $2 \mathrm{H} 11$ enhances the binding of SWII-binding small molecules to KRAS ${ }^{\mathrm{WT}}$. KRAS ${ }^{\mathrm{WT}}$ immobilized on an SPR chip and injected with concentrations ranging from 1 to $50 \mu \mathrm{M}$ in the absence or presence of $2 \mathrm{H} 11$.

(B) (Upper panel) X-ray crystal structure of $2 \mathrm{H} 11 \mathrm{Fab}$ bound to KRAS ${ }^{\mathrm{G} 12 \mathrm{C}}$-GDP. Rendering is as follows: KRAS (grey ribbons), SWI (teal color), SWII (dark blue color), GDP (sticks), and $\mathrm{Mg}^{2+}$ (sphere). Cys12 sidechain is highlighted by thicker sticks. 2H11 Fab is shown in transparent surface and ribbons (heavy chain is green and light chain is brown). (Lower panel) Surface mapping of KRAS epitope for 2H11 in which heavy chain and light chain contacts are colored green and orange, respectively.

(C) A close-up view of the 2H11:KRAS ${ }^{\mathrm{G} 12 \mathrm{C}}$ interface. Complementarity-determining regions (CDRs) making direct contact with KRAS are shown in ribbons, the central Trp99 is shown in thick sticks, and hydrogen bonds are indicated by red dotted lines.

(D) Comparison of KRAS ${ }^{\mathrm{G} 12 \mathrm{C}}: 2 \mathrm{H} 11$ complex in the presence (orange ribbon) and absence (grey, blue, teal ribbons) of GNE-1952. The compound is shown in sticks with carbon atoms in yellow. The SWII residues of both structures are shown in thin sticks. 
(E) ELISA experiments with both 1A5 and 2H11 CLAMPS show binding to a panel of KRAS-GDP mutants indicating a common epitope $(n=3)$. 


\section{Supplement Figures}

\section{Fig. S1: Additional characterization of CLAMPs.}

(A) Structures of KRAS G12C covalent small molecules.

(B) Representative SPR traces of $1 \mathrm{~A} 5$ and $2 \mathrm{H} 11$ against KRAS ${ }^{\mathrm{G} 12 \mathrm{C}}-\mathrm{GDP}+\mathrm{GNE}-1952$ and KRAS ${ }^{\mathrm{G} 12 \mathrm{C}}-\mathrm{GDP}$.

(C) Epitope binning results indicate that group 1 and group bind two overlapping epitopes.

\section{Fig. S2: Cell-based assays with 1A5 CLAMP.}

(A) No observable KRAS staining by 1A5 CLAMP in HCT116 KRAS ${ }^{G 13 D}$ cells treated with a KRAS ${ }^{G 12 C}$ inhibitor GNE-1952.

10 (B) Immunoblot analysis for covalently modified KRAS and KRAS pathway markers (pERK, pMEK, and pS6) in a bulk population of HCC1171 KRAS ${ }^{G 12 C}$ mutant cancer cells. Washout experiments reveals that KRAS ${ }^{\mathrm{G} 12 \mathrm{C}}$ covalent modification is maintained for 24 hours after removal of ARS-853.

\section{Fig. S3: Biophysical and structural analysis of $2 \mathrm{H11}$ CLAMP.}

15 (A) $2 \mathrm{H} 11$ enhances the binding of SWII pocket ligands to KRAS ${ }^{\mathrm{WT}}$ and KRAS ${ }^{\mathrm{G} 12 \mathrm{C}}$. (B) Structural alignment of 2H11 CLAMP bound to KRAS ${ }^{\mathrm{G} 12 \mathrm{C}}$-GDP and DCAI ligand bound to KRAS (PDB 4DST). KRAS ${ }^{\mathrm{G} 12 \mathrm{C}}$ is depicted as white ribbons with SWII highlighted in blue. 2H11 heavy chain is colored in green, and Trp99 rendered in sticks.

DCAI bound KRAS ${ }^{\mathrm{G} 12 \mathrm{D}}$ is colored in red, with DCAI shown rendered in sticks. DCAI and Trp99 bind to the same site on KRAS.

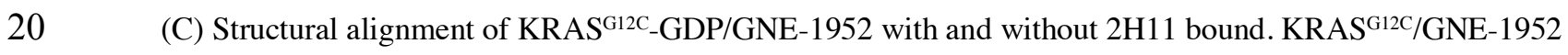
without $2 \mathrm{H} 11$ is colored in cyan. KRAS ${ }^{\mathrm{G} 12 \mathrm{C}} / \mathrm{GNE}-1952$ with $2 \mathrm{H} 11$ is colored in orange, compound is colored in yellow, and 2H11 heavy chain is colored in green.

(D) Structural comparison between iDab (orange, PDB 2UZI) and 2H11 (green) in SWI (teal) binding suggest less sensitivity to different nucleotide states for $2 \mathrm{H} 11$. 
Table S1.

Affinity of CLAMPs for a variety of KRAS-GDP proteins.

\begin{tabular}{|c|c|c|c|c|c|}
\hline & $\begin{array}{c}\text { KRAS }^{\text {G12C-GDP + }} \\
\text { GNE-1952 }\end{array}$ & $\begin{array}{c}\text { KRAS }^{\mathrm{G}_{12} \mathrm{C}_{-} \text {GDP + }} \\
\text { ARS-853 }\end{array}$ & $\begin{array}{c}\text { KRAS }^{\mathrm{G}_{12} \mathrm{C}_{-} \mathrm{GDP}} \\
+ \text { +ARS-1620 }\end{array}$ & 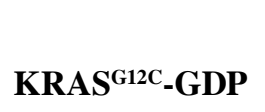 & KRAS $^{\text {WT_GDP }}$ \\
\hline Clone & $\begin{array}{c}\text { Affinity } \\
\text { (nM) }\end{array}$ & $\begin{array}{c}\text { Affinity } \\
\text { (nM) }\end{array}$ & $\begin{array}{c}\text { Affinity } \\
\text { (nM) }\end{array}$ & $\begin{array}{c}\text { Affinity } \\
\text { (nM) }\end{array}$ & $\begin{array}{c}\text { Affinity } \\
\text { (nM) }\end{array}$ \\
\hline 1D6 & 10 & NB & NB & NB & NB \\
\hline 1B7 & 139 & NB & NB & NB & NB \\
\hline $2 \mathrm{C} 1$ & 7 & NB & NB & NB & NB \\
\hline $1 \mathrm{~A} 6$ & 61 & NB & NB & NB & NB \\
\hline $1 \mathrm{~F} 4$ & 39 & NB & NB & NB & NB \\
\hline $1 \mathrm{~A} 5$ & 2 & 4 & 2 & 513 & 159 \\
\hline $1 \mathrm{E} 5$ & 5 & NB & 107 & 1700 & 411 \\
\hline $2 \mathrm{~A} 3$ & 10 & 195 & 21 & 216 & 71 \\
\hline $2 \mathrm{H} 11$ & 5 & 4 & 54 & 230 & 42 \\
\hline $3 \mathrm{~A} 12$ & 2 & 772 & 94 & 121 & 24 \\
\hline $4 \mathrm{G} 12$ & 1 & 441 & NB & 366 & 61 \\
\hline
\end{tabular}


Table S2.

Crystallographic data collection and refinement statistics.

\begin{tabular}{|c|c|c|c|}
\hline & KRAS $^{\text {G12C/GNE-1952 }}$ & $\mathrm{KRAS}^{\mathrm{G} 12 \mathrm{C}} / 2 \mathrm{H} 11$ & KRAS $^{\text {G12C/GNE-1952/2H11 }}$ \\
\hline PDB code & 6XC6 & $6 \mathrm{XC5}$ & $6 \mathrm{XC8}$ \\
\hline Space group & $\mathrm{P} 1$ & $\mathrm{C} 2$ & $\mathrm{P} 2_{1}$ \\
\hline Unit cell & $\begin{array}{l}a=33.6 \AA, b=44.0 \AA, \\
c=65.3 \AA, \\
\alpha=89.0^{\circ}, \beta=85.0^{\circ}, \gamma=80.0^{\circ}\end{array}$ & $\begin{array}{l}a=149.9 \AA, b=68.8 \AA, \\
c=101.0 \AA, \\
\alpha=\gamma=90^{\circ}, \beta=114.1^{\circ}\end{array}$ & $\begin{array}{l}a=59.2 \AA, b=51.9 \AA, c=107.5 \AA, \\
\alpha=\gamma=90^{\circ}, \beta=131.0^{\circ}\end{array}$ \\
\hline Resolution & $2.15 \AA$ & $2.20 \AA$ & $2.00 \AA$ \\
\hline $\begin{array}{l}\text { Total measured } \\
\text { reflections }\end{array}$ & $69746(639)^{1}$ & $317770(48051)^{1}$ & $151279(22570)^{1}$ \\
\hline $\begin{array}{l}\text { Completeness } \\
(\%)\end{array}$ & $89.3(90.5)$ & $99.8(100)$ & $98.1(98.9)$ \\
\hline Redundancy & $3.9(3.9)$ & $6.7(6.9)$ & $3.5(3.5)$ \\
\hline $\mathrm{I} / \sigma$ & $8.4(2.1)$ & $11.6(1.8)$ & $15.5(2.9)$ \\
\hline Rsym $^{2}$ & $0.098(0.540)$ & $0.068(0.892)$ & $0.038(0.362)$ \\
\hline $\mathrm{CC}_{1 / 2}$ & $0.995(0.854)$ & $0.999(0.816)$ & $0.999(0.886)$ \\
\hline \multicolumn{4}{|l|}{ Refinement } \\
\hline Resolution range & $50-2.15 \AA$ & $50-2.20 \AA$ & $50-2.00 \AA$ \\
\hline Rcryst $^{3} /$ Rfree $^{4}$ & $0.213 / 0.253$ & $0.208 / 0.225$ & $0.191 / 0.234$ \\
\hline $\begin{array}{l}\text { Non-hydrogen } \\
\text { atoms }\end{array}$ & 2978 & 4788 & 4902 \\
\hline Water molecules & 148 & 104 & 290 \\
\hline Average B & $39.4 \AA^{2}$ & $79.7 \AA^{2}$ & $51.4 \AA^{2}$ \\
\hline $\begin{array}{l}\text { r.m.s.d. bond } \\
\text { lengths }\end{array}$ & $0.002 \AA$ & $0.004 \AA$ & $0.008 \AA$ \\
\hline r.m.s.d. angles & $0.497^{\circ}$ & $0.991^{\circ}$ & $0.952^{\circ}$ \\
\hline Ramachandran $^{5}$ & $0.928 / 0.065 / 0.007 / 0$ & $0.883 / 0.110 / 0.002 / 0.006$ & $0.920 / 0.074 / 0 / 0.006$ \\
\hline
\end{tabular}

$5 \quad{ }^{1}$ Values in parentheses are of the highest resolution shell

${ }^{2} \mathrm{Rsym}=\Sigma \mathrm{I}_{\mathrm{hi}}-\mathrm{I}_{\mathrm{h}} / / \Sigma \mathrm{I}_{\mathrm{hi}}$, where $\mathrm{I}_{\mathrm{hi}}$ is the scaled intensity of the $i$ th symmetry-related observation of reflection $h$ and $\mathrm{I}_{\mathrm{h}}$ is the mean value.

${ }^{3}$ Rcryst $=\Sigma_{h} \mid F_{\text {oh }}-F_{c h} / / \Sigma_{h} F_{o h}$, where $F_{o h}$ and $F_{c h}$ are the observed and calculated structure factor amplitudes for reflection $h$.

$10 \quad{ }^{4}$ Value of Rfree is calculated for 5\% randomly chosen reflections not included in the refinement.

${ }^{5}$ Values following Ramachandran indication fraction of residues fall into most favored/additional allowed/generally allowed/disallowed regions of Ramachandran plot. 


\section{Materials and Methods}

KRAS $^{\text {G12C }}$ small molecules

GNE-1952, ARS-853, ARS-1620, AMG510 were synthesized as reported in the literature. Non-covalent GNE-1952 was prepared using the following synthesis procedure:

1-(4-(6-chloro-7-(5-methyl-1H-indazol-4-yl)quinazolin-4-yl)piperazin-1-yl)ethanone

Synthetic Scheme

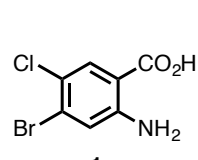

1

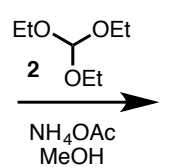

$\mathrm{MeOH}$
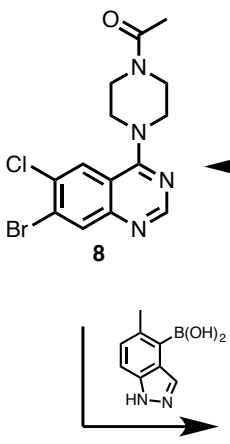
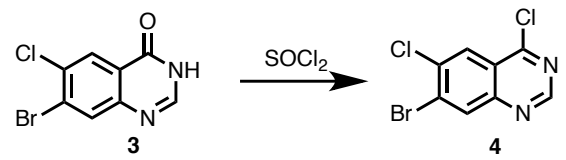

4
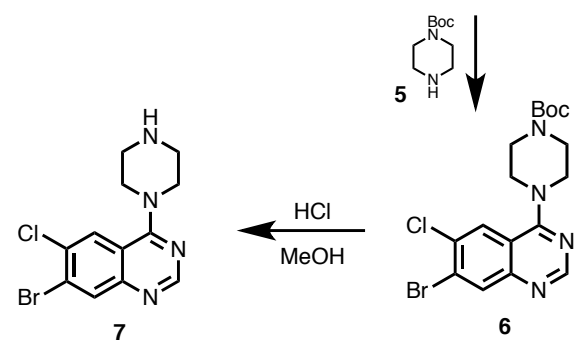

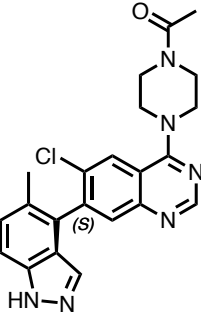

S-9

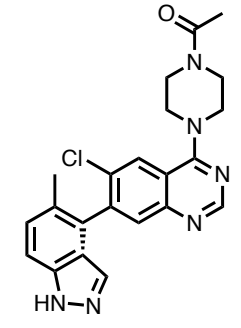

$R-9$

\section{Synthesis of 7-bromo-6-chloroquinazolin-4(3H)-one 3}

A mixture of 2-amino-4-bromo-5-chlorobenzoic acid 1 (9.00 g, $35.9 \mathrm{mmol})$, triethoxymethane $\mathbf{2}(27.7 \mathrm{~g}, 359 \mathrm{mmol})$ and $\mathrm{NH}_{4} \mathrm{OAc}(60.0 \mathrm{~mL}, 359 \mathrm{mmol})$ in $20 \mathrm{~mL}$ of $\mathrm{MeOH}$ was heated at $120^{\circ} \mathrm{C}$ overnight. Upon cooling, the resulting solid was filtered and washed with cold $\mathrm{MeOH}(2 \times 20 \mathrm{~mL})$ to afford 7-bromo-6-chloroquinazolin-4(3H)-one 3 as a yellow solid in $81 \%$ yield $(8.5 \mathrm{~g})$.

\section{Synthesis of tert-butyl 4-(7-bromo-6-chloroquinazolin-4-yl)piperazine-1-carboxylate 6}

To a solution of 7-bromo-6-chloroquinazolin-4(3H)-one 3 (8.50 g, $32.9 \mathrm{mmol})$ in $50 \mathrm{~mL}$ of $\mathrm{SOCl}_{2}$ was added DMF

( 3 drops). The resulting mixture was refluxed for 2 hours. After removal of $\mathrm{SOCl}_{2}$, the residue was dissolved in 200 $\mathrm{mL}$ of dioxane, followed by the addition of DIPEA $(17.0 \mathrm{~mL}, 98.7 \mathrm{mmol})$ and tert-butyl piperazine-1-carboxylate 5 $(9.20 \mathrm{~g}, 49.3 \mathrm{mmol})$. The mixture was stirred at $110^{\circ} \mathrm{C}$ for 2 hours. Upon cooling, the reaction was diluted with water $(100 \mathrm{~mL})$ and extracted with EtOAc $(3 \times 100 \mathrm{~mL})$. The organic layers were combined, washed with brine $(200 \mathrm{~mL})$, and dried over $\mathrm{Na}_{2} \mathrm{SO}_{4}$. The mixture was concentrated and the residue was stirred in $100 \mathrm{~mL}$ of $\mathrm{MeOH}$ to afford a 6 in $81 \%$ yield $(11.3 \mathrm{~g})$ 


\section{Synthesis of 1-(4-(7-bromo-6-chloroquinazolin-4-yl)piperazin-1-yl)ethanone 8}

To a solution of tert-butyl 4-(7-bromo-6-chloroquinazolin-4-yl)piperazine-1-carboxylate 6 (1.00 g, $2.34 \mathrm{mmol})$ in $\mathrm{MeOH} / \mathrm{DCM}(10 \mathrm{~mL} / 10 \mathrm{~mL})$ was added $15 \mathrm{~N} \mathrm{HCl}$ in $\mathrm{MeOH}(5 \mathrm{~mL})$ at $0{ }^{\circ} \mathrm{C}$. The mixture was stirred at $0^{\circ} \mathrm{C}$ for 1 hour, then r.t. for 2 hours. Upon removal of solvent, the residue was combbined with DCM (20 mL), followed by the addition of $\mathrm{Et}_{3} \mathrm{~N}(0.96 \mathrm{~mL}, 7.02 \mathrm{mmol})$ and acetyl chloride $8(0.20 \mathrm{~mL}, 2.57 \mathrm{mmol})$ at $0{ }^{\circ} \mathrm{C}$. The resulting mixture was diluted with EtOAc $(30 \mathrm{~mL})$, washed with water $(25 \mathrm{~mL})$, and brine $(25 \mathrm{~mL})$. The organic solution was dried over $\mathrm{Na}_{2} \mathrm{SO}_{4}$. After removal of solvent, the residue was purified by column chromatography eluted with $2.5 \% \mathrm{MeOH}$ in DCM to afford 1-(4-(7-bromo-6-chloroquinazolin-4-yl)piperazin-1-yl)ethanone 9 as a light brown solid in 77\% yield $(0.66 \mathrm{~g})$.

Synthesis of 1-(4-(6-chloro-7-(5-methyl-1H-indazol-4-yl)quinazolin-4-yl)piperazin-1-yl)ethanone 9

A mixture of 1-(4-(7-bromo-6-chloroquinazolin-4-yl)piperazin-1-yl)ethanone 8 (200 mg, $0.543 \mathrm{mmol}$ ), 5-methyl-1Hindazol-4-yl-4-boronic acid (287 mg, $1.63 \mathrm{mmol})$ and $\mathrm{Na}_{2} \mathrm{CO}_{3}(0.23 \mathrm{~g}, 2.17 \mathrm{mmol})$ in a sealed tube with dioxane/water $(8 \mathrm{~mL} / 2 \mathrm{~mL})$ was purged with $\mathrm{N}_{2}$ for $10 \mathrm{~min}$. After addition of $\mathrm{Pd}\left(\mathrm{PPh}_{3}\right)_{4}(63 \mathrm{mg}, 0.054 \mathrm{mmol})$, the mixture was purged with $\mathrm{N}_{2}$ for another $10 \mathrm{~min}$. The resulted mixture was sealed and heated at $100{ }^{\circ} \mathrm{C}$ overnight. The reaction was cooled and mixed with $30 \mathrm{~mL}$ of water, then extracted with EtOAc $(3 \times 30 \mathrm{~mL})$. The combined organic solution was dried over $\mathrm{Na}_{2} \mathrm{SO}_{4}$. After removal of solvent, the residue was purified by column chromatography eluted with $2.5-5 \%$ $\mathrm{MeOH}$ in DCM to afford 1-(4-(6-chloro-7-(5-methyl-1H-indazol-4-yl)quinazolin-4-yl)piperazin-1-yl)ethanone as a yellow solid in 53\% yield $(120 \mathrm{mg})$. The resulting solids were further purified by chiral SFC to afford the desired atropisomers $S \mathbf{- 9}$ and $R-\mathbf{9}$.

\section{Phage selections}

Selections were performed using existing synthetic Fab phage display libraries ${ }^{34,35}$. The pooled library was cycled through three to four rounds of binding in solution to biotinylated KRAS ${ }^{\mathrm{G} 12 \mathrm{C}}$-GDP+GNE-1952 (concentrations started at $500 \mathrm{nM}$ and were gradually decreased to $10 \mathrm{nM}$ in the last round). The solution was captured on neutravidin beads (Promega), blocked with $5 \mu \mathrm{M}$ biotin, washed three times for $30 \mathrm{~s}$ each in PBS $+0.5 \% \mathrm{BSA}+$ $0.1 \%$ Tween 20 (PBSBT), and eluted with $100 \mathrm{mM} \mathrm{HCl}$. The eluted phage was neutralized with $1 \mathrm{M}$ TRIS-HCl $\mathrm{pH}$ 8.0 prior to overnight amplification in E. coli XL1-blue (Stratagene) with the addition of M13KO7 helper phage (New England Biolabs). In order to enrich for binders specific to the covalently modified KRAS ${ }^{\mathrm{G} 12 \mathrm{C}}$, selections were done in the presence of excess of either soluble KRAS ${ }^{\mathrm{G} 12 \mathrm{C}}$-GDP or KRAS ${ }^{\mathrm{G} 12 \mathrm{C}}$-GMPPCP at $1 \mu \mathrm{M}$. After selections, individual colonies were picked and grown overnight at $30^{\circ} \mathrm{C}$ in 96 -well deep well plates in 2xYT media supplemented with carbenicillin and helper phage. Phage supernatants were used in phage ELISAs against $\mathrm{KRAS}^{\mathrm{G} 12 \mathrm{C}}$-GDP+GNE1952, KRAS ${ }^{\mathrm{G} 12 \mathrm{C}}$-GDP, and KRAS ${ }^{\mathrm{G} 12 \mathrm{C}}$-GMPPCP to identify target-specific clones.

\section{Antibody and Fab production}

35 Sequences from lead phage clones were obtained by Sanger sequencing. IgG (human IgG1) expression constructs for the light chain and heavy chain for each clone were obtained by gene synthesis (Genscript, South San Francisco, 
CA). IgGs were expressed by transient transfection of 293 cells and purified with affinity chromatography followed by SEC using standard methods (MabSelect SuRe; GE Healthcare, Piscataway, NJ, USA).

Fab constructs for bacterial expression were generated by gene synthesis (Genscript, South San Francisco, CA). Recombinant Fab was generated as previously described ${ }^{36}$.

\section{Antibody ELISA against covalently modified KRAS ${ }^{\mathrm{G} 12 \mathrm{C}}$}

Biotinylated KRAS ${ }^{\mathrm{G} 12 \mathrm{C}}$-GDP+GNE-1952 and KRAS ${ }^{\mathrm{G} 12 \mathrm{C}}$-GDP were coated on neutravidin ELISA plates (Thermo Scientific) in triplicate at $0.3 \mu \mathrm{g} / \mathrm{mL}$ in PBS and incubated overnight at $4^{\circ} \mathrm{C}$. Plates were washed with PBSBT and serial dilutions of anti-KRAS antibodies (both in-house and commercial) starting at $10 \mu \mathrm{g} / \mathrm{mL}$ were added and shaken for $1-2$ hours at $25^{\circ} \mathrm{C}$. After washing, a species matched Fc-specific HRP $2^{\circ}$ antibody (1:10,000 dilution) was added and shaken for 1 hour at $25^{\circ} \mathrm{C}$. After washing with PBSBT, plates were developed with TMB substrate for 5 minutes and detected at $650 \mathrm{~nm}$. The commercial antibodies used were as follows: iDab6 ${ }^{27}$ as a rabbit IgG, anti-Ras antibody (EP1125Y) (Abcam, ab52939), KRAS-2B specific Rabbit polyclonal (Proteintech, Cat. No 16155-1-AP), Ras10 (Millipore, Cat. No 05-516), 3B10-2F2 (Sigma-Aldrich, Cat. No WH0003845M1), and 234-4.2 (Millipore, Cat. No OP24).

\section{Antibody ELISA against mutant KRAS-GDP proteins}

KRAS-GDP proteins were directly coated in triplicate at $10 \mu \mathrm{g} / \mathrm{mL}$ on Maxisorb plates (Thermo Scientific) in PBS and incubated overnight at $4^{\circ} \mathrm{C}$. Plates were blocked for 2 hours at $25^{\circ} \mathrm{C}$ using $4 \%$ BSA. Serial dilutions of 1 A5 and $2 \mathrm{H} 11$ antibodies starting at $10 \mu \mathrm{g} / \mathrm{mL}$ were added for $1-2$ hours at $25^{\circ} \mathrm{C}$ with shaking. Plates were developed and read as described above.

\section{Antibody surface plasmon resonance (SPR)}

SPR experiments were carried out on the Mass- 1 (Bruker) at $25^{\circ} \mathrm{C}$ using HBS-P+ (GE Healthcare) running buffer. $1 \mu \mathrm{g} / \mathrm{mL}$ of the anti-KRAS antibodies were captured using an anti-HuIgG1 Fc capture kit (GE Healthcare). KRAS $^{\mathrm{G} 12}$-GDP+GNE-1952, KRAS $^{\mathrm{G} 12 \mathrm{C}}$ _GDP, KRAS ${ }^{\mathrm{G} 12 \mathrm{C}}$-GDP+ARS-1620, KRAS $^{\mathrm{G} 12 \mathrm{C}}$-GDP+ARS-853, and KRAS $^{\text {WT }}{ }_{\text {GGDP }}$ were added as analytes in solution at a flow rate of $30 \mu \mathrm{L} / \mathrm{min}$. KRAS ${ }^{\mathrm{G} 12}$-GDP+GNE-1952 was titrated using a dilution series from 500-0 nM. KRAS ${ }^{\mathrm{G} 12 \mathrm{C}_{-}} \mathrm{GDP}$ was titrated using a dilution series from 5000-0 $\mathrm{nM}$.

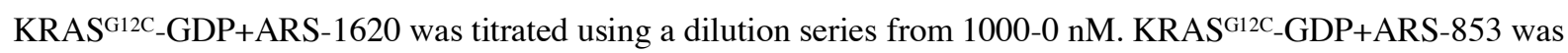
titrated using a dilution series from 200-0 nM. KRAS ${ }^{\text {WT }}$-GDP was titrated using a dilution series from 2000-0 nM. Sensorgrams were fit to a 1:1 Langmuir model to identify kinetic parameters.

\section{Epitope binning}

Epitope binning experiments were performed in HBS-P+ (GE Healthcare) running buffer at $25^{\circ} \mathrm{C}$ on an array-based imager (IBIS MX96, Netherlands, as described previously ${ }^{37}$. Briefly, $10 \mu \mathrm{g} / \mathrm{mL}$ of anti-KRAS antibody was amine coupled onto surface in $10 \mathrm{mM}$ sodium acetate $\mathrm{pH} 4.5$ and the surface was quenched with $1 \mathrm{M}$ ethanolamine. Epitope 
binning experiments were done by initially flowing $2 \mu \mathrm{M} \mathrm{KRAS}^{\mathrm{G} 12 \mathrm{C}}-\mathrm{GDP}+\mathrm{GNE}-1952$ over the immobilized antibodies and flowing over $10 \mu \mathrm{g} / \mathrm{mL}$ of each of the anti-KRAS antibodies in solution. Enough time was allowed for association of the antigen prior to the addition of the antibody. Prior to the addition of the next antibody in solution, the surface was regenerated with $10 \mathrm{mM}$ glycine $\mathrm{pH} 2.5$.

\section{Immunofluorescence and High Content Imaging}

Cells (20000 to 40000 cells per well depending on the cell line) were seeded into Poly-L-Lysine Coated 96-well plates (Cell Carrier Ultra; Perkin Elmer) and supplemented with complete medium (RPMI with 2\% L-Glutamine and $10 \% \mathrm{FBS}$ ). The next day, cells were treated with $\mathrm{KRAS}^{\mathrm{G} 12 \mathrm{C}}$ inhibitors at indicated concentrations and incubated for the indicated length of time. At the end of treatment, cells were washed twice with cold 1XPBS, fixed with 3\% paraformaldehyde for $20 \mathrm{~min}$ at $25^{\circ} \mathrm{C}$, washed for $10 \mathrm{~min}$ with $1 \mathrm{X} \mathrm{PBS}$, and the PFA was quenched with 50mM $\mathrm{NH}_{4} \mathrm{Cl}$ for $10 \mathrm{~min}$ at $25^{\circ} \mathrm{C}$. Cells were washed twice with $1 \mathrm{X}$ PBS for 5 min and then permeabilized with $1 \mathrm{X}$ Perm/Wash Buffer (BD, Fisher Scientific) for $20 \mathrm{~min}$ at $25^{\circ} \mathrm{C}$. Cells were then incubated with primary antibody (1A5 or iDab or both) diluted in Perm/Wash buffer at indicated concentration for 2 hours at $25^{\circ} \mathrm{C}$. Cells were then washed three times with Perm/Wash buffer for $10 \mathrm{~min}$ each, and the incubated with conjugated fluorescence secondary antibody (1:500; Alexa488 anti-human and Alexa647 anti-rabbit or anti-rat at 1:500 from Jackson ImmunoResearch Laboratories Inc.) for 20 to $60.100 \mathrm{ml}$ of 300nM DAPI was added to each well for $15 \mathrm{~min}$ followed by two washes with Perm/Wash buffer, and one wash with 1XPBS prior to imaging. Imaging was done on the Opera Phenix ${ }^{\mathrm{TM}}$ HCS machine (PerkinElmer Inc.) using the 40X water immersion lens and the confocal mode for better membrane scanning ability. Four or five fields were acquired for each well to enable better quantitative analysis of fluorescence intensities, and analysis and quantification were conducted on the Harmony® (PerkinElmer Inc.) software and plotted using GraphPad Prism.

For washout experiments, cells were plated as described above and treated with KRAS ${ }^{\mathrm{G} 12 \mathrm{C}}$ inhibitor and incubated with compound for $18-24 \mathrm{hrs}$. One plate was imaged after $24 \mathrm{hrs}$ as control and the other plates were washed twice with cold 1X PBS. Plates were then incubated for either 24 or $48 \mathrm{hrs}$ with $150 \mathrm{~mL}$ of complete compound-free medium and stained and imaged as described above.

For pERK rebound studies, cell were NCI-H358 cells were seeded at cell density of 6000 cells in Poly-D-Lysine coated Cell Carrier-96 Ultra Microplates (PerkinElmer). The next day, cells were treated with KRAS ${ }^{\mathrm{G} 12 \mathrm{C}}$ inhibitors at indicated concentrations and incubated for the indicated length of time. Cells were fixed with $4 \%$ paraformaldehyde (PFA), then permeabilized with ice-cold $100 \%$ methanol for $10 \mathrm{~min}$ at $-20{ }^{\circ} \mathrm{C}$ followed by a $1 \mathrm{X}$ PBS wash for $5 \mathrm{~min}$. Cells were then co-stained with $0.25 \mathrm{ug} / \mathrm{ml} 1 \mathrm{~A} 5$ and 1:800 dilution of pERK (Cell Signaling Technology, Cat\# 4370S) followed by staining with conjugated fluorescence secondary antibodies (Alexa488 donkey anti-human at 1:500 and Alexa647 donkey anti-rabbit at 1:500 from Jackson ImmunoResearch Laboratories Inc.) at room temperature for $60 \mathrm{~min}$. 


\section{Immunoblot studies}

HCC1171 cells (20000 per mL) were seeded into T-75 ultra low adherence ULA plates (Corning ${ }^{\circledR}$ Inc.) with complete medium (RPMI with 2\% L-Glutamine and 10\% FBS) and allowed to grow overnight. The next day, cells were treated with $5 \mu \mathrm{M}$ ARS-853 for 18-24 hrs. The next day cells were pelleted and washed twice with 1X PBS and replenished with compound-free complete medium with or without $50 \mathrm{mg} / \mathrm{mL}$ Cycloheximide (Sigma) as a control for new protein synthesis for 24 or 48 hours. Cells were then collected for the end of treatment, washed once with 1X PBS, and lysed with Ripa Buffer (Thermofisher Scientific ${ }^{\mathrm{TM}}$ ) with Halt ${ }^{\mathrm{TM}}$ proteases and phosphatases inhibitors (Thermofisher Scientific ${ }^{\mathrm{TM}}$ ) to collect protein. Pierce ${ }^{\mathrm{TM}}$ BCA assay (Thermofisher Scientific ${ }^{\mathrm{TM}}$ ) was used to quantify protein content. Lysates were then run on Novex ${ }^{\mathrm{TM}} 4-20 \%$ Tris-Glycine gels for $3 \mathrm{hrs}$ at $100 \mathrm{~V}$, and

10 transferred using the Trans-Blot ${ }^{\circledR}$ Turbo ${ }^{\mathrm{TM}}$ Transfer System (Bio-Rad Laboratories). Membranes were blocked with Li-Cor Odyssey® TBS blocking buffer for $1 \mathrm{hr}$, incubated with primary antibodies (Proteintech: KRAS antibody \#12063-1-AP, Cell Signaling Technology: pERK(Thr 202/Tyr 204) \#9101, Total ERK \#9102, pS6 (Ser 235/236) \#2211, and HSP90 \#4874) overnight, then washed three times with TBST for before adding the secondary antibodies (Li-Cor). Membranes were finally washed three times with TBST buffer and imaged on the Li-Cor

15 Odyssey ${ }^{\circledR}$ CLx machine.

\section{In vitro flow cytometry experiments}

HCC1171 cells were plated in 6 well plate overnight, then treated with a dose range of ARS-853 for 24hrs. Cells were collected by trypsin and fixed with 500ul BD Cytofix Buffer (BD\# 554655) at room temperature for 20min. Cells were pelleted and washed with $3 \mathrm{ml}$ BD Perm/wash buffer (BD\#554723). Cells were stained with 1st

20 antibody-conjugate in BD Perm/wash buffer, around 0.5ug antibody/100ul total cell for 2 hours at 4 degree. Antibodies used are:1A5-647 (inhouse, conjugated with Invitrogen\#A20186); pHH3-488 (CST\#9708); pS6V450 (BD\#561457). After staining, cells were washed with 3ml BD Perm/wash buffer one time then suspended in 250ul PI stain buffer (0.1\% Triton X-100, 0.1\% NaCitrate, 0.1nM EDTA, 1mM Tris-pH7.5) with 50ug/ml PI, 50ug/ml RNase A, run on LSRII_C.

\section{Immunoprecipitation studies}

HCC1171 cells were treated with DMSO or 5uM ARS-1620 for 24hours. Cells were collected and lysed in IP lysis buffer (Thermo 87787) with addition of 300mM NaCl, protease inhibitors (Roche11836170001) and phosphatase inhibitors (Roche 4906845001). 1ug of antibody was mixed with 200ug lysate in 100ul IP buffer, incubated on ice for 1hour. 15ul proteinA/G MAG bead in 200ul IP lysis buffer was added to each antibody/lysate mix, and rotated at 4 degree overnight. Beads were washed 3 times with IP lysis buffer, then suspended in 1x LDS loading buffer and boiled at 95 degree for 10min. Supernatant were loaded on gels for Western blot. 


\section{In vivo tumor studies}

All procedures were approved by and conformed to the guidelines and principles set by the Institutional Animal Care and Use Committee of Genentech and were carried out in an Association for the Assessment and Accreditation of Laboratory Animal Care (AAALAC)-accredited facility. Female C.B-17 SCID (Inbred) mice that were 16-17 weeks old and weighed 24-27 g were obtained from Charles River Lab. Mice were inoculated with five million NCI-H358 non-small cell lung carcinoma cells (suspended in a 1:1 mixture of Hank's Balanced Salt Solution containing Matrigel at a 1:1 ratio) in both the left and right flank subcutaneously. Tumors were monitored until they reached a mean tumor volume of $400-600 \mathrm{~mm}^{3}$. Mice were given single dose of 0 (Vehicle $-100 \%$ Labrasol), 50, or $200 \mathrm{mg} / \mathrm{kg}$ ARS-1620 orally (PO) by gavage in a volume of $100 \mu \mathrm{L}$. Plasma and tumor samples were collected at 8 or 24 hours post-dose.

\section{Flow cytometry assays on tumor samples}

To evaluate tumor pharmacodynamics, harvested tumors were digested with Liberase DL $(0.2 \mathrm{U} / \mathrm{ml}$, Sigma-Aldrich) and DNase I (40 U/ml, Sigma-Aldrich) for $30 \mathrm{~min}$ at $37^{\circ} \mathrm{C}$ using gentleMACS ${ }^{\mathrm{TM}}$ dissociator

15 (Miltenyi Biotec). Single cell suspensions were prepared and stained for EpCAM (clone EBA1, BD Biosciences) and Fixable Viability Dye (ebioscience) for 30 minutes at $4^{\circ} \mathrm{C}$ and washed. Cells were fixed with Cytofix Buffer (BD Biosciences) for 30 minutes at $4^{\circ} \mathrm{C}$ and washed with Perm/Wash buffer (BD Biosciences). Intracellular staining was performed for Alexa488-labeled 1A5 and pS6 (Clone N7-548, BD Biosciences) for 60 minutes at $4^{\circ} \mathrm{C}$. Samples were then washed with perm wash buffer and resuspended in FACS buffer. Cells were

20 analyzed by FACS on the BD Symphony instrument. Data were analyzed using GraphPad prism software version 7 (GraphPad, San Diego, CA) and Flowjo 10.5.3 (FlowJo, BD, CA).

\section{KRAS $^{G 12 C}$ protein expression and purification}

The N-terminal His-tagged KRAS ${ }^{\mathrm{G} 12 \mathrm{C}}$ (1-169) constructs with and without cysteine mutations (S39C, C51S, C80L, C118S) were cloned into pET-52b vector and transformed into BL21(DE3) cells. Cells were grown at $37^{\circ} \mathrm{C}$ to an OD600 absorbance of $0.5 \mathrm{in} \mathrm{LB}$ media containing $50 \mu \mathrm{g} / \mathrm{mL}$ of carbenicillin and then transferred to $16^{\circ} \mathrm{C}$ prior to induction with $0.3 \mathrm{mM}$ IPTG at an OD600 absorbance of 0.8. Cells were harvested 16 hours post induction and the pellet was lysed by passing through a microfluidizer in a buffer containing $50 \mathrm{mM}$ Hepes pH 8.0, $500 \mathrm{mM}$ $\mathrm{NaCl}, 5 \mathrm{mM} \mathrm{MgCl} 2,10 \mathrm{mM}$ Imidazole, 10\% Glycerol, $1 \mathrm{mM}$ TCEP, $1 \mathrm{mM}$ PMSF, benzonase and EDTA-freeprotease inhibitors. Cell lysates were clarified by centrifugation at $12,000 \mathrm{rpm}$ for 1 hour. Clarified cell lysates were loaded onto a HiTrap column in a buffer containing $20 \mathrm{mM}$ Hepes $\mathrm{pH}$ 8.0, $300 \mathrm{mM} \mathrm{NaCl}, 10 \%$ Glycerol, $5 \mathrm{mM}$ $\mathrm{MgCl} 2,1 \mathrm{mM}$ TCEP and bound KRAS protein was eluted with $300 \mathrm{mM}$ Imidazole. The N-terminal His-tag was 
cleaved by incubating with TEV protease and removed through a nickel column. The KRAS protein was polished by a size-exclusion S75 column (GE Healthcare) in a buffer of $20 \mathrm{mM}$ Hepes pH 8.0, $150 \mathrm{mM} \mathrm{NaCl}, 5 \mathrm{mM} \mathrm{MgCl}$. The purity of KRAS was greater that $95 \%$ as assayed by SDS-PAGE. To load GDP on KRAS, it was first incubated with $40 \mathrm{mM}$ EDTA and $2 \mathrm{mM}$ GDP at $20^{\circ} \mathrm{C}$ for 1-2 hours. It was then buffer exchanged to an EDTA-free and nucleotide-free buffer.

\section{KRAS $^{\text {G12C }}$ protein covalent modification by GNE-1952}

To covalently modify KRAS with GNE-1952, KRAS ${ }^{\mathrm{G} 12 \mathrm{C}}$ was incubated overnight at $20^{\circ} \mathrm{C}$ with $5 \mathrm{mM}$ GDP, $20 \mathrm{mM}$ EDTA and $150 \mu \mathrm{M}$ GNE-1952 in a buffer of $20 \mathrm{mM}$ Hepes pH 8.0, $150 \mathrm{mM} \mathrm{NaCl}, 10 \%$ glycerol and $2 \mathrm{mM}$ TCEP. Complete covalent modification was confirmed by observing shift in mass via mass spectrometry. KRAS was buffer exchanged by a size-exclusion S75 16/60 column into a buffer of $20 \mathrm{mM}$ Hepes pH 7.0, $150 \mathrm{mM}$ $\mathrm{NaCl}$ and $10 \%$ glycerol.

\section{Crystallization of $\operatorname{KRAS}^{\mathrm{G} 12 \mathrm{C}}$ and $2 \mathrm{H11}$ Fab}

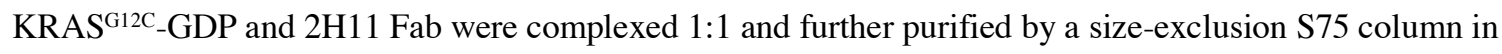

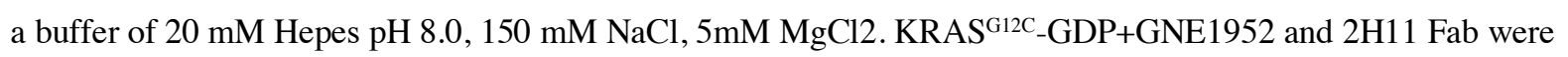
complexed 1:1 and further purified by size-exclusion S75 column in a buffer of $20 \mathrm{mM}$ Hepes pH 8.0, $150 \mathrm{mM}$ $\mathrm{NaCl}$. Diffraction quality crystals of $\mathrm{KRAS}^{\mathrm{G} 12 \mathrm{C}} / 2 \mathrm{H} 11$ were grown at $19^{\circ} \mathrm{C}$ from $1.0 \mu \mathrm{L}+1.0 \mu \mathrm{L}$ vapor diffusion sitting drops containing $10 \mathrm{mg} / \mathrm{mL}$ KRAS and $24 \mathrm{mg} / \mathrm{mL} 2 \mathrm{H} 11 \mathrm{Fab}$ against a crystallization buffer of $0.1 \mathrm{M}$ sodium cacodylate pH 6.5, 40\% 2-methyl 2,4-pentanediol (MPD), 7\% PEG 8000, 0.5\% ethyl acetate, $10 \mathrm{mM}$ spermine tetrahydrochloride. Crystals appeared in two weeks and typically grew to $150 \times 20 \times 30 \mu \mathrm{M}$

Diffraction quality crystals of KRAS ${ }^{\mathrm{G} 12 \mathrm{C}}-\mathrm{GDP} / \mathrm{GNE}-1952 / 2 \mathrm{H} 11$ were grown at $19^{\circ} \mathrm{C}$ from $1.0 \mu \mathrm{L}+1.0 \mu \mathrm{L}$ vapor diffusion sitting drops containing $15 \mathrm{mg} / \mathrm{mL}$ of KRAS/2H11 complex against a crystallization buffer of $0.1 \mathrm{M}$ MES pH 6.0,21\% PEG 4K and 0.2 M lithium sulfate. Crystals appeared in 10 days and grew to a size of $100 \times 15$ x $30 \mu \mathrm{M}$.

$\mathrm{KRAS}^{\mathrm{G} 12 \mathrm{C}}$-GDP/GNE-1952 crystals were grown at $19^{\circ} \mathrm{C}$ from $1.0 \mu \mathrm{L}+1.0 \mu \mathrm{L}$ vapor diffusion sitting drops containing KRAS ${ }^{\mathrm{G} 12 \mathrm{C}}$-GDP/GNE-1952 against a crystallization buffer of $0.10 \%$ n-Octyl-B-D-glucoside, $0.1 \mathrm{M}$ sodium citrate $\mathrm{pH} 5.5,22 \%$ PEG 3350. To prepare for diffraction data collection, $10 \%$ glycerol was added to the crystallization buffer as cryobuffer before flash freezing the crystals for above three cases.

\section{Diffraction data collection and structure determination}

The diffraction data of KRAS ${ }^{\mathrm{G} 12 \mathrm{C}} / \mathrm{GNE}-1952$, KRAS $^{\mathrm{G} 12 \mathrm{C}} / 2 \mathrm{H} 11$, and KRAS ${ }^{\mathrm{G} 12 C} / \mathrm{GNE}-1952 / 2 \mathrm{H} 11$ crystals were collected using monochromatic X-rays at Stanford Synchrotron Radiation Lightsource (SSRL) beamline 12-2 
or Advanced Light Source (ALS) beam line 5.0.2 using PILATUS3 6M detector. Rotation method was applied to a single crystal for each of the complete data set. The crystals were kept at cryogenic temperature throughout the data collection process. Data reduction was performed using the program XDS ${ }^{38}$ and the CCP4 program suites ${ }^{39}$. Data reduction statistics are shown in Table S2.

The structures were phased by molecular replacement (MR) using program Phaser ${ }^{39}$. A previously published crystal structure of KRAS ${ }^{\mathrm{G} 12 \mathrm{D}}$ (PDB code 4DSU) and a Fab structure (PDB code 3R1G) were used as the MR search models. Manual rebuilding was performed with graphics program COOT ${ }^{40}$. The structures were further refined iteratively using program REFMAC5 ${ }^{41}$ and PHENIX ${ }^{42}$ using maximum likelihood target functions, anisotropic individual B-factor refinement and TLS refinement, and to achieve final statistics shown in Supplementary Table 2 .

\section{Immunohistochemistry studies}

Frozen OCT- embedded tumor samples were sectioned $5 \mu \mathrm{m}$ thick using a cryostat, and acetone-fixed at $25^{\circ} \mathrm{C}$. Prior to IHC staining, sections were air-dried overnight at $25^{\circ} \mathrm{C}$. Endogenous peroxidase activity was quenched with glucose oxidase solution for 60 minutes at $37^{\circ} \mathrm{C}$. Sections were loaded onto the BOND III autostainer and then incubated with the $1 \mathrm{~A} 5 \mathrm{CLAMP}$, diluted to $3 \mu \mathrm{g} / \mathrm{ml}$ in $3 \% \mathrm{BSA} / \mathrm{PBS}$ for 60 minutes at $37^{\circ} \mathrm{C}$. Sections were then incubated in rabbit anti-Human IgG, (Jackson ImmunoResearch, 309-005-082) diluted to $5 \mu \mathrm{g} / \mathrm{mL}$ in $3 \%$ BSA/PBS, for 30-minutes, followed by detection with PowerVision Poly-HRP anti-rabbit (Leica, PV6119) for 30 minutes. Sections were incubated in Metal enhanced DAB (Thermofisher scientific, 34065), then counterstained

20 with Mayer's Hematoxylin (Rowley Biochemical, L-756-1A) for $1 \mathrm{~min}$, followed by incubation in Richard-Allen Scientific Bluing Reagent (Thermo Fisher Scientific, 7301) for $1 \mathrm{~min}$, and finally dehydrated in graded reagent alcohol. Sections were coverslipped with Tissue-Tek Glas Mounting Medium (Sakura, 6419).

\section{SPR Experiments with 2H11 CLAMP and small molecules}

A series S SA (streptavidin) chip was inserted into a Biacore T200 (GE Health Sciences). The instrument was primed into running buffer (50 mM HEPES pH 7.5, $150 \mathrm{mM} \mathrm{NaCl}, 0.2 \%$ (w/v) PEG-3350, 0.1\% CM-dextran (w/v), $0.1 \mathrm{mM}$ TCEP, $10 \mathrm{mM} \mathrm{MgCl} 2,100 \mathrm{nM} \mathrm{GDP}$, and 2\% (v/v) DMSO). KRAS ${ }^{\mathrm{G} 12 \mathrm{C}}$ pre-blocked at the 12 position with a covalent inhibitor was captured to yield 2000-2500 RU on flow channel 1 (FC1) and FC3 to serve as the reference for $\mathrm{KRAS}^{\mathrm{WT}}$ and $\mathrm{KRAS}^{\mathrm{G} 12 \mathrm{C}}$ and allow affinity measurement exclusively at the Switch II pocket. $\mathrm{KRAS}^{\mathrm{WT}}$ or KRAS ${ }^{\mathrm{G} 12 \mathrm{C}}$ was captured on FC2 and FC4 within $100 \mathrm{RU}$ of the reference channel capture level and data was collected in FC 2-1, FC 4-3 mode. All channels were subsequently blocked by injecting $100 \mu \mathrm{g} / \mathrm{mL}$ aminePEG-biotin (Thermo Fisher). 2H11 was injected 2 times at $200 \mathrm{nM}$ for 120 seconds at the start of the run to saturate FC3 and FC4, and injected every 14 cycles at $100 \mathrm{nM}$ throughout the run to ensure saturation throughout the run. Analyte samples were tested $503 \mathrm{M}-1.753 \mathrm{M}$ in 2-fold dose response with 20-30 second contact time and 30 second dissociation. Data was analyzed in with a 1:1 affinity model in Biacore S200 Evaluation Software 1.0 and figures were made in Scrubber 2 (Biologic Software). 
Figure 1

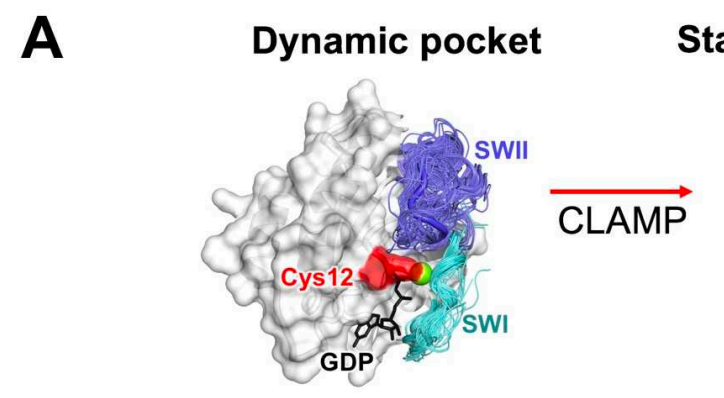

Stabilized "open" pocket

\section{B}
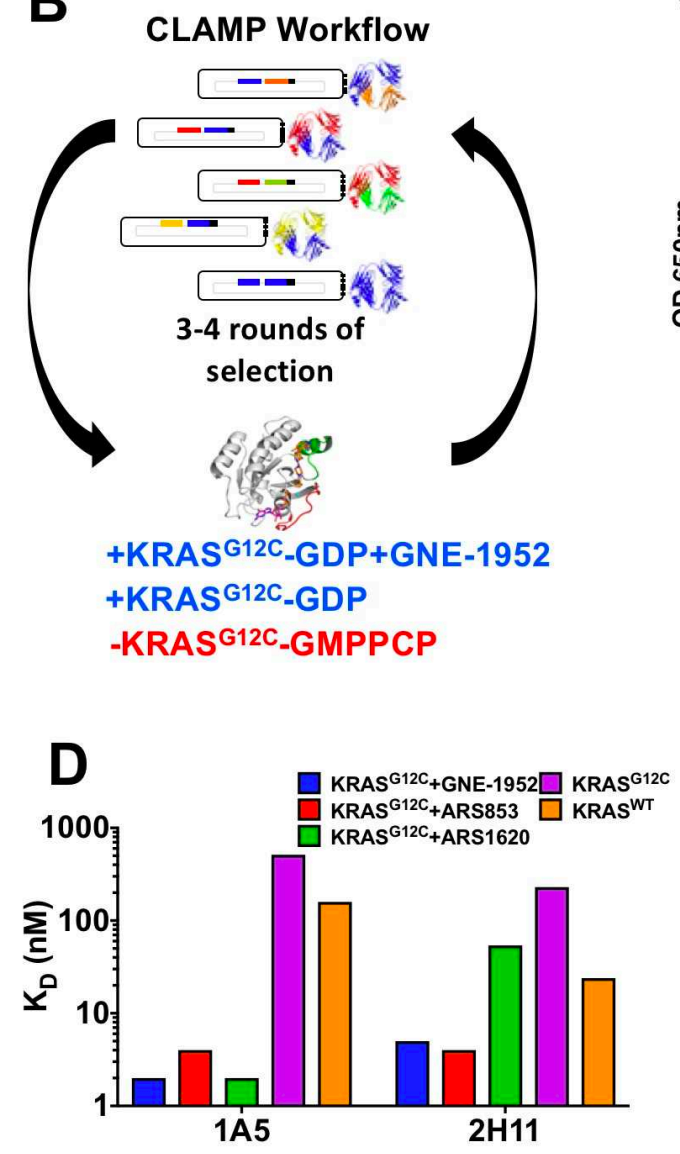

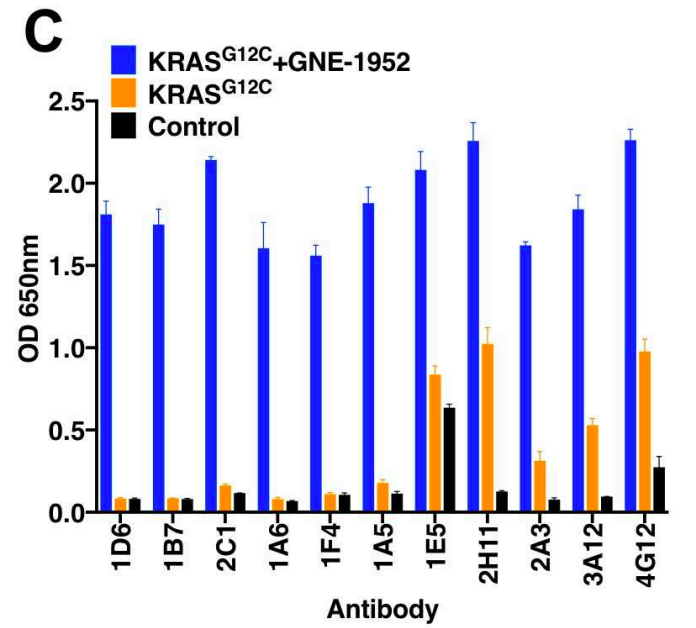

E
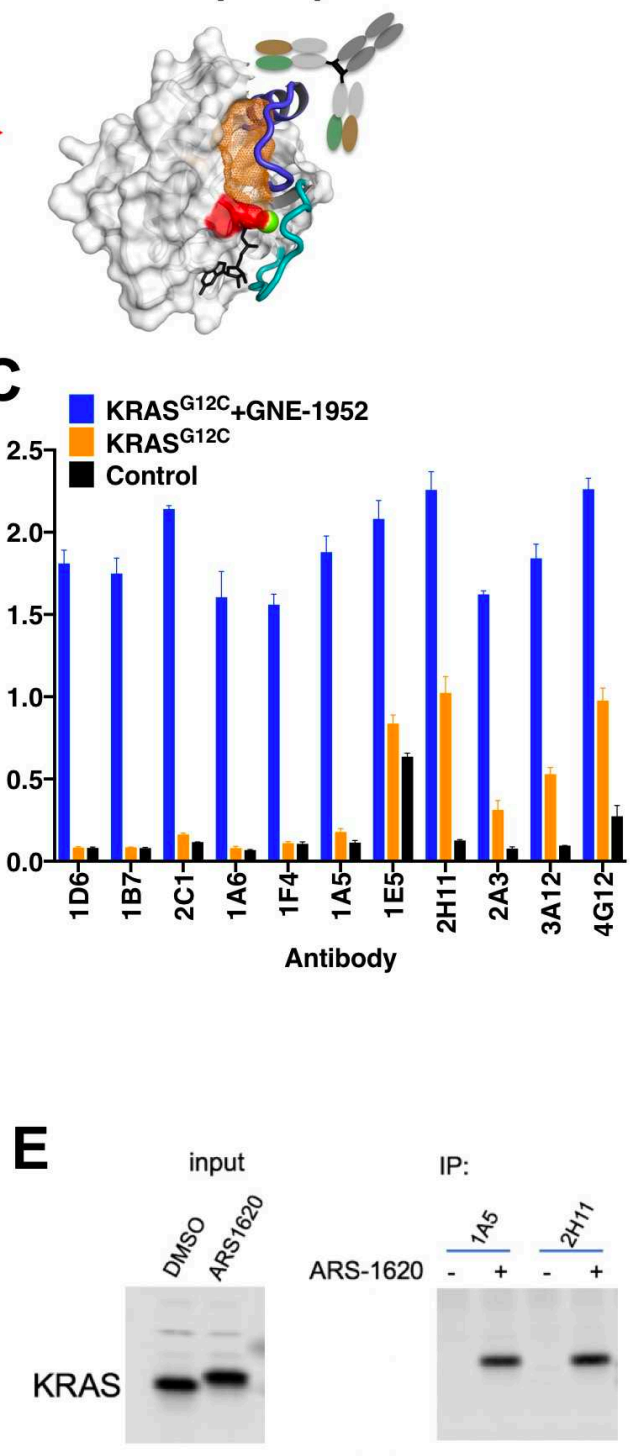
Figure 2

\section{A}

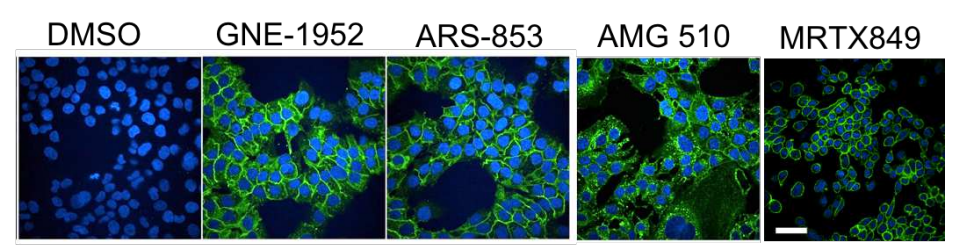

B
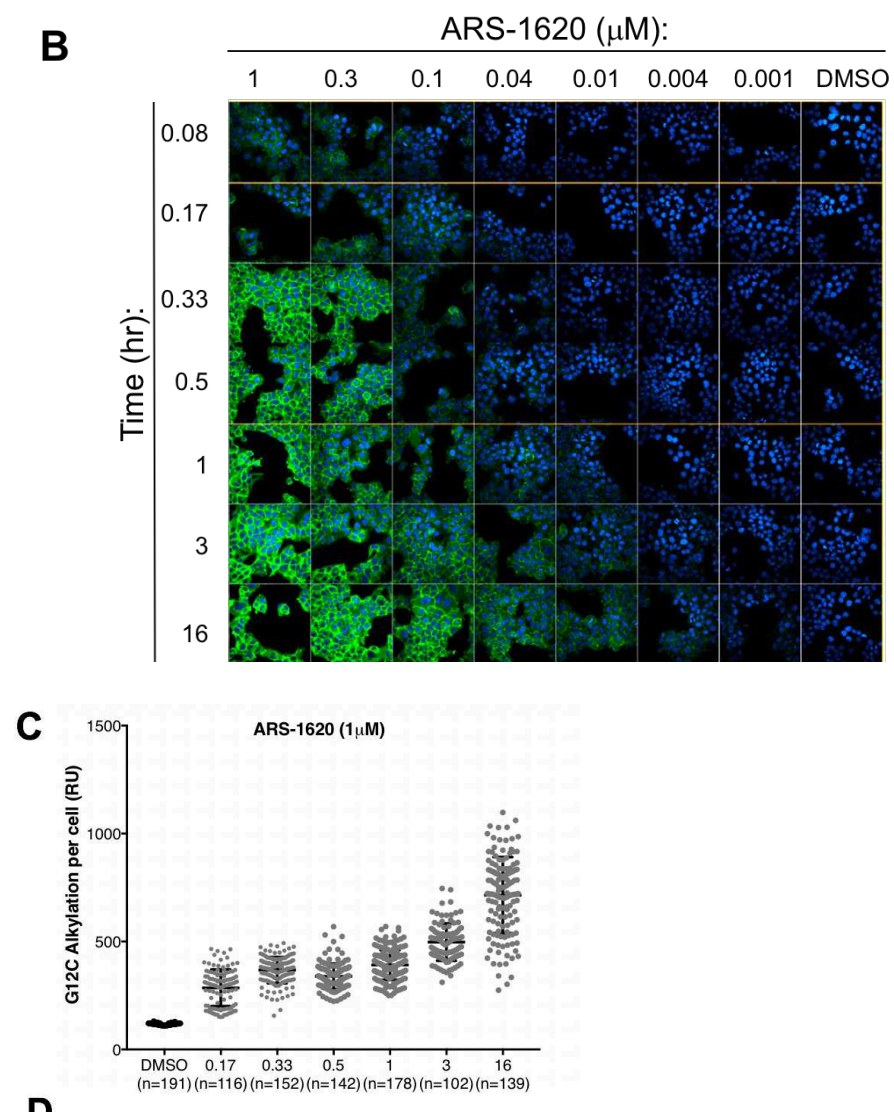

D
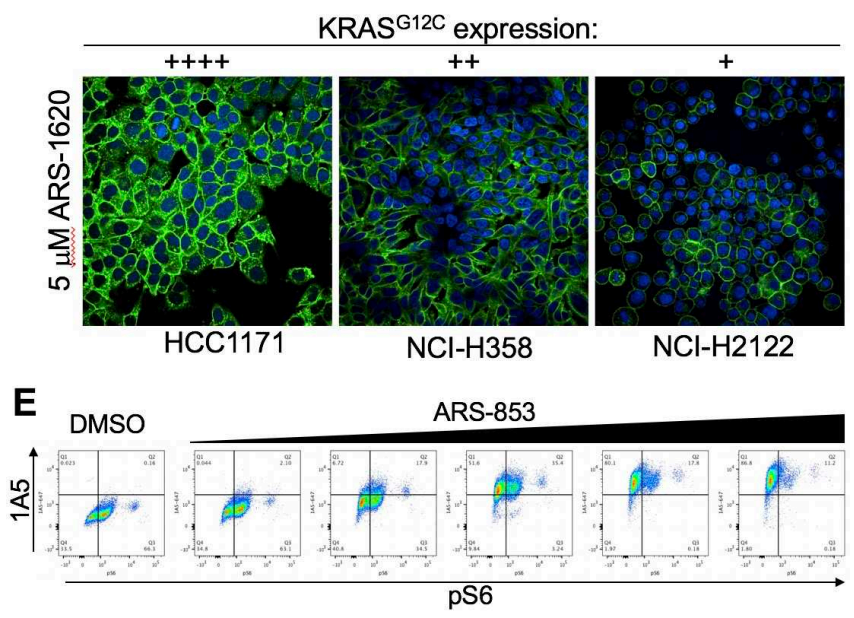
Figure 3
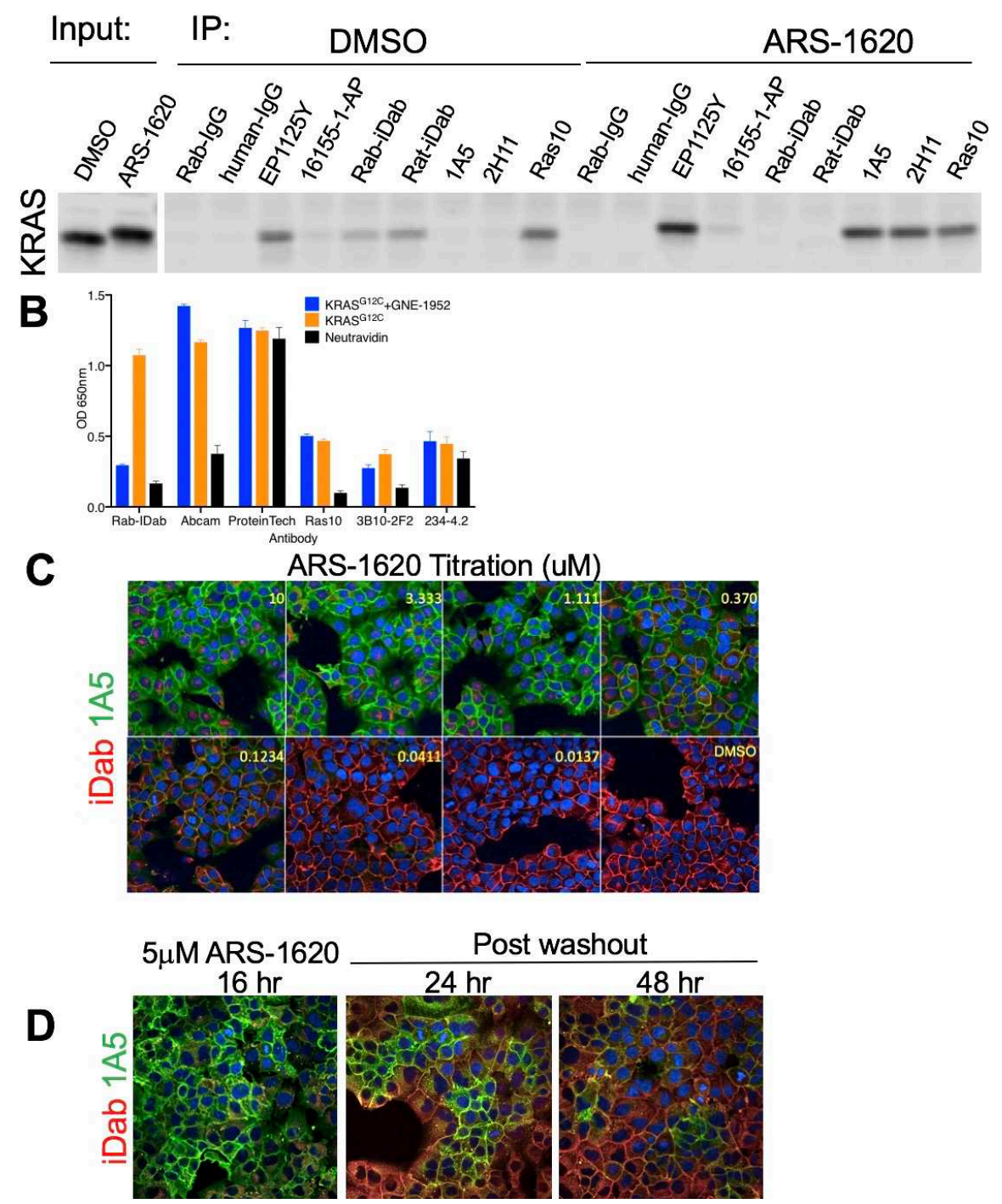
Figure 3 (continued)

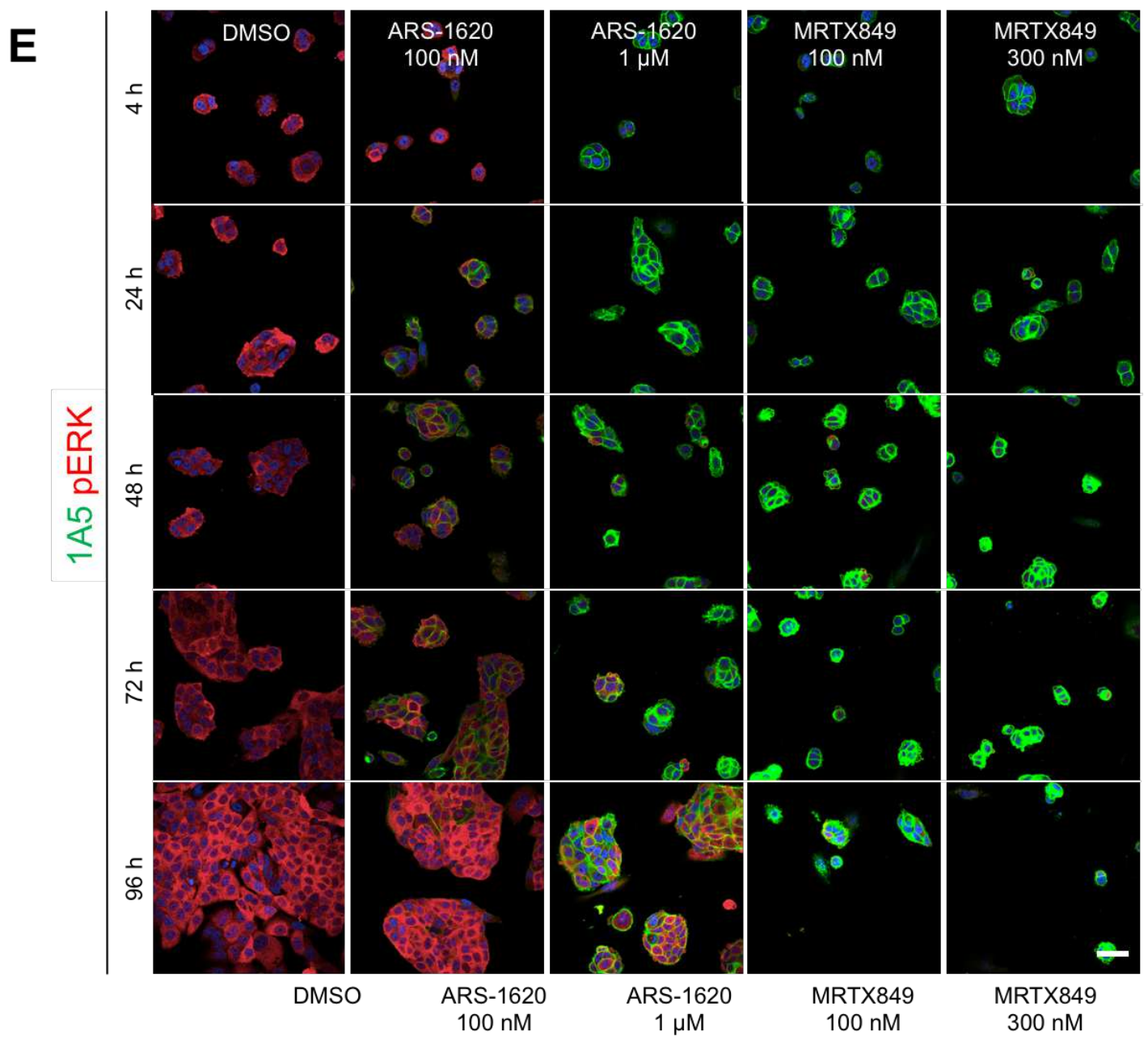


Figure 4
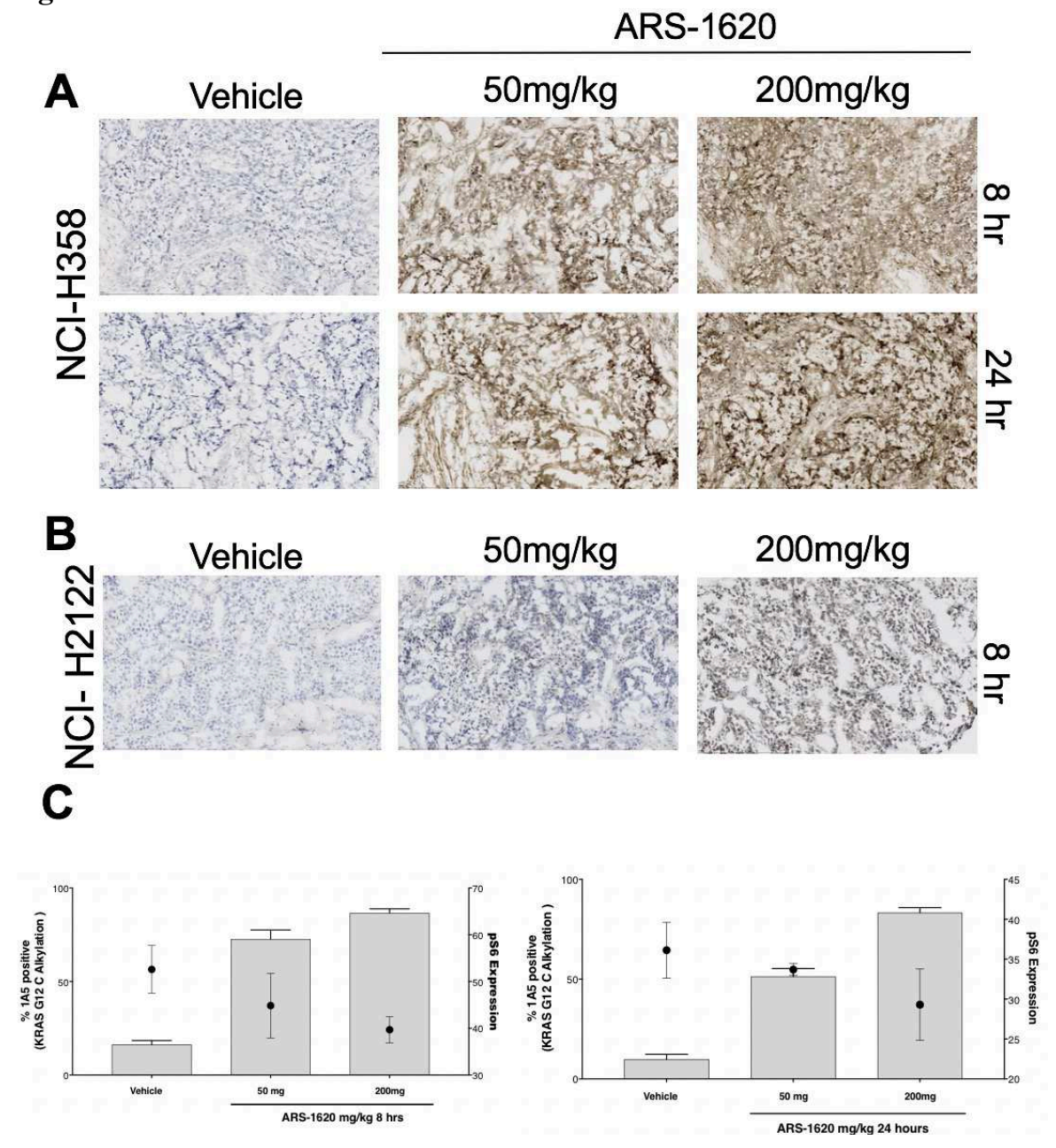
Figure 5
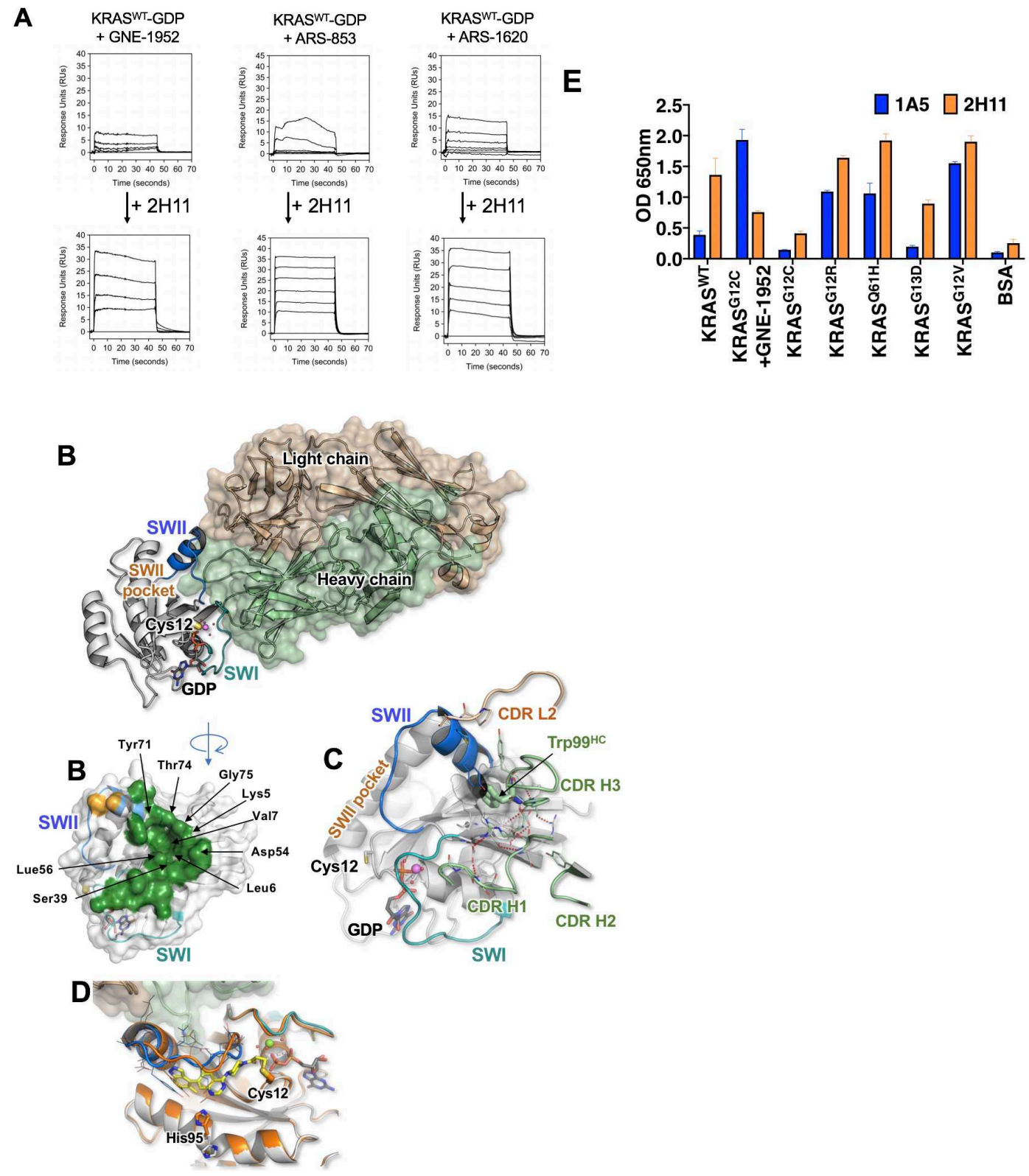


\section{Supplement Figures}

Fig. S1.

A

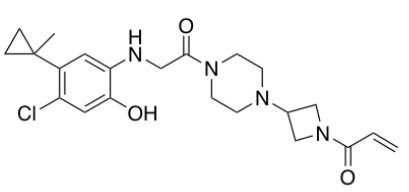

ARS-853

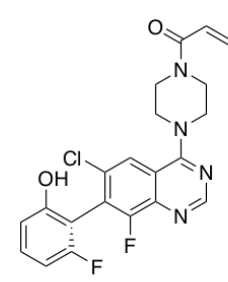

ARS-1620

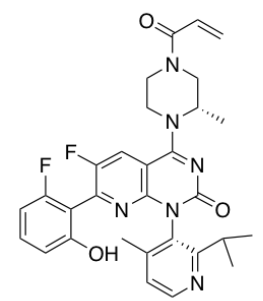

AMG 510

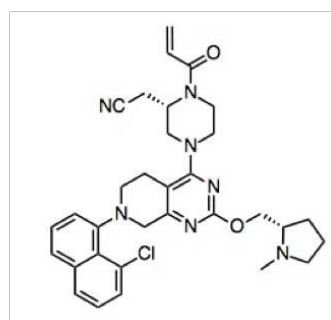

MRTX849

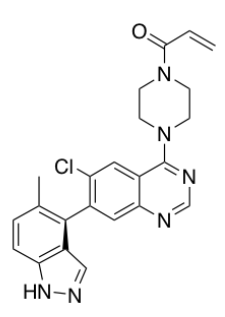

GNE-1952

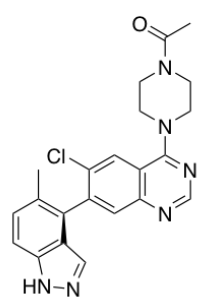

Non-covalent GNE-1952

B
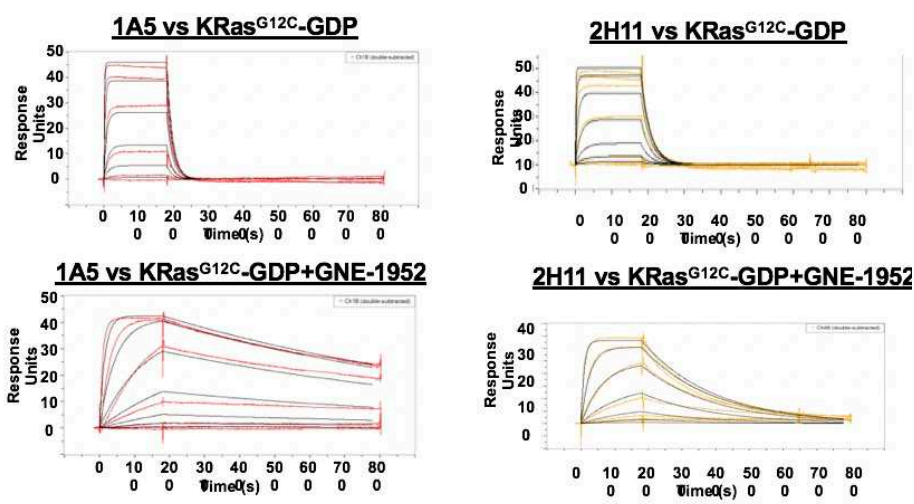

2H11 vs KRas ${ }^{G 12 C-G D P+G N E-1952}$

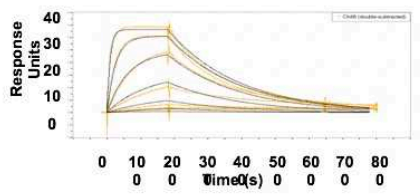

C

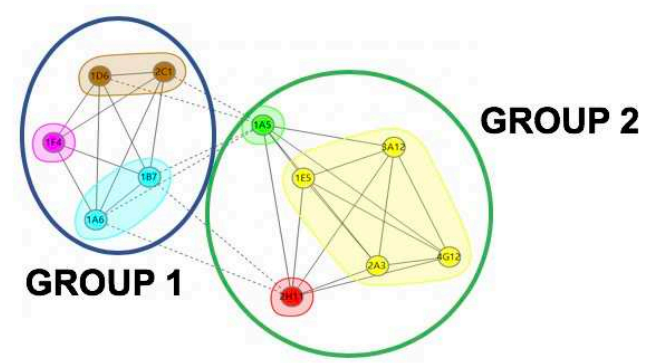


Fig. S2.

A

HCT116 (KRAS G13D)

KRAS expression:

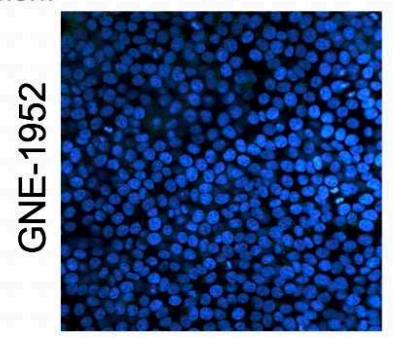

B

\begin{tabular}{|c|c|c|c|c|c|c|c|c|c|c|c|}
\hline & & & \multicolumn{9}{|c|}{ Washout } \\
\hline & & & $6 \mathrm{hr}$ & & 24 & & & & 48 & & \\
\hline DMSO & + & - & - & + & + & - & - & + & + & - & - \\
\hline 5mM ARS-853 & - & + & + & - & - & + & + & - & - & + & + \\
\hline ug/ml Cycloheximide & - & - & $-\quad+$ & - & + & - & + & - & + & - & + \\
\hline
\end{tabular}

Covalently modified KRAS ${ }^{\mathrm{G} 12 \mathrm{C}} \rightarrow-$ Unmodified KRAS ${ }^{\mathrm{G} 12 \mathrm{C}}$

$\mathrm{pERK}===\equiv=\overline{=}=$

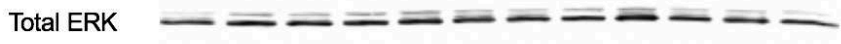

pS6 $\quad-\quad-\cdots-\cdots$

HSP90 - - - - - - - - - 
Fig. S3.

A KRASG12C_GDP KRas

KRASWt_GDP KRas

KRAS ${ }^{\mathrm{G} 12 \mathrm{C}}$-GDP KRas

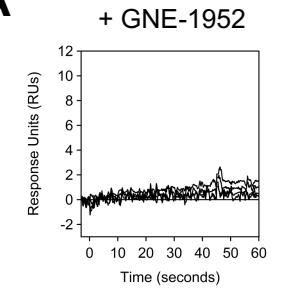

$\downarrow+2 \mathrm{H} 11$

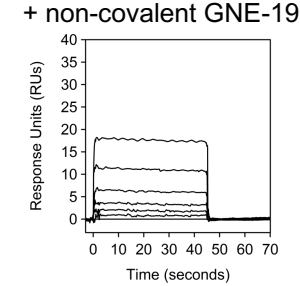

+ non-covalent GNE-1952
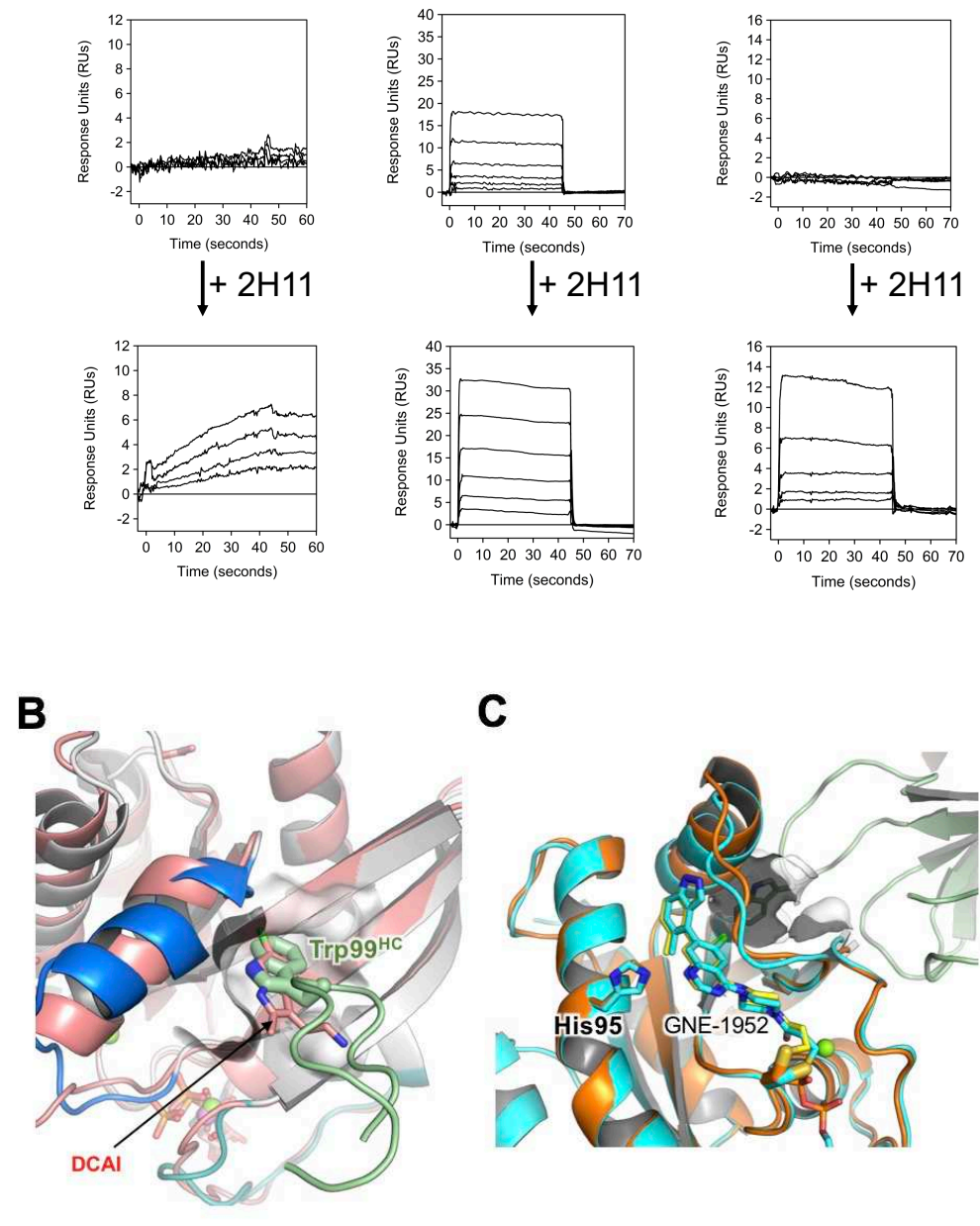

C
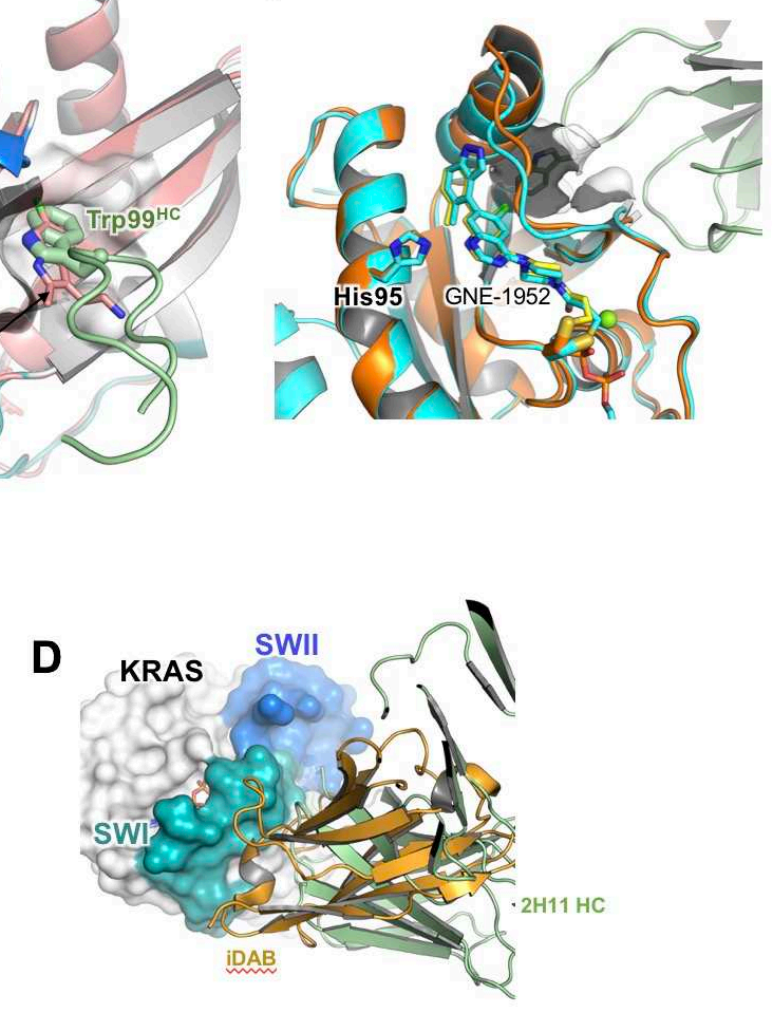